\title{
Breaking the 1/2-barrier for the twisted second moment of Dirichlet L-functions
}

DOI:

10.1016/j.aim.2020.107175

\section{Document Version}

Accepted author manuscript

Link to publication record in Manchester Research Explorer

\section{Citation for published version (APA):}

Bui, H., Pratt, K., Robles, N., \& Zaharescu, A. (2020). Breaking the 1/2-barrier for the twisted second moment of Dirichlet L-functions. Advances in Mathematics, 370, [107175]. https://doi.org/10.1016/j.aim.2020.107175

\section{Published in:}

Advances in Mathematics

\section{Citing this paper}

Please note that where the full-text provided on Manchester Research Explorer is the Author Accepted Manuscript or Proof version this may differ from the final Published version. If citing, it is advised that you check and use the publisher's definitive version.

\section{General rights}

Copyright and moral rights for the publications made accessible in the Research Explorer are retained by the authors and/or other copyright owners and it is a condition of accessing publications that users recognise and abide by the legal requirements associated with these rights.

\section{Takedown policy}

If you believe that this document breaches copyright please refer to the University of Manchester's Takedown Procedures [http://man.ac.uk/04Y6Bo] or contact uml.scholarlycommunications@manchester.ac.uk providing relevant details, so we can investigate your claim.

\section{OPEN ACCESS}




\title{
BREAKING THE $\frac{1}{2}$-BARRIER FOR THE TWISTED SECOND MOMENT OF DIRICHLET $L$-FUNCTIONS
}

\author{
HUNG M. BUI, KYLE PRATT, NICOLAS ROBLES AND ALEXANDRU ZAHARESCU
}

\begin{abstract}
We study the second moment of Dirichlet $L$-functions to a large prime modulus $q$ twisted by the square of an arbitrary Dirichlet polynomial. We break the $\frac{1}{2}$-barrier in this problem, and obtain an asymptotic formula provided that the length of the Dirichlet polynomial is less than $q^{51 / 101}=q^{1 / 2+1 / 202}$. As an application, we obtain an upper bound of the correct order of magnitude for the third moment of Dirichlet $L$-functions. We give further results when the coefficients of the Dirichlet polynomial are more specialized.
\end{abstract}

\section{INTRODUCTION}

We study the mean square of the product of Dirichlet $L$-functions with arbitrary Dirichlet polynomials. The central problem is to obtain an asymptotic formula for

$$
\sum_{\chi(\bmod q)}^{*}\left|L\left(\frac{1}{2}, \chi\right)\right|^{2}\left|\sum_{a \leq q^{\kappa}} \frac{\boldsymbol{\alpha}_{a} \chi(a)}{\sqrt{a}}\right|^{2},
$$

where $\sum^{*}$ denotes summation over all primitive characters $\chi$ modulo $q$, the coefficients $\boldsymbol{\alpha}_{a} \ll a^{\varepsilon}$ are arbitrary, and $0<\kappa<1$. The asymptotic evaluation when $\kappa<1 / 2$ was established by Iwaniec and Sarnak [14]. In this regime the main term comes from the "diagonal" contribution, and the off-diagonal terms contribute only to the error.

In this work we obtain an asymptotic expression for (1.1) with $\kappa$ going beyond the $\frac{1}{2}$-barrier. In this larger regime some off-diagonal terms contribute to the main term, and evaluating this contribution, as well as bounding the error terms, is considerably more difficult. Duke, Friedlander and Iwaniec [5] proved that the quantity in (1.1) may be bounded by $O_{\varepsilon}\left(q^{1+\varepsilon}\right)$ for some $\kappa>1 / 2$, but their proof does not extend to give an asymptotic formula. Very recently, Conrey, Iwaniec and Soundararajan [4] applied their asymptotic large sieve and studied (1.1) with an additional averaging over the modulus $q$.

The analogous problem to (1.1) for the Riemann zeta-function was studied by Bettin, Chandee and Radziwiłł [2], who broke the $\frac{1}{2}$-barrier for an arbitrary Dirichlet polynomial. Our work is inspired by their beautiful paper, but there are significant differences between the family of primitive Dirichlet $L$-functions in the $q$-aspect and the Riemann zeta-function in the $t$-aspect. These differences usually make the family of Dirichlet $L$-functions more difficult to work with. In fact, Bettin, Chandee and Radziwiłl mentioned the work of Duke, Friedlander and Iwaniec [5], and said: "Our proof of Theorem 1 would not extend to give an asymptotic in this case, and additional input is needed."

2010 Mathematics Subject Classification. 11M06, $11 \mathrm{M} 26$.

Key words and phrases. Dirichlet $L$-functions, twisted second moment, moments, Kloosterman fractions, large sieve inequality. 
It turns out that it is more convenient to work with a more general version of (1.1) by introducing "shifts". We let $\alpha, \beta \in \mathbb{C}$ satisfy $\operatorname{Re}(\alpha), \operatorname{Re}(\beta) \ll(\log q)^{-1}$ and, for our later application ${ }^{1}, \operatorname{Im}(\alpha), \operatorname{Im}(\beta) \ll \log q$. Furthermore, we treat the characters $\chi$ according to their parity to ensure that the $L$-functions under consideration have the same gamma factors in their functional equations. Let $\varphi^{+}(q)$ be the number of even primitive characters $\chi$ modulo $q$, and let $\sum^{+}$denote summation over all these characters. In this work we deal exclusively with even Dirichlet characters, but our arguments go through identically for odd characters. For fixed $0<\kappa<1$, we study

$$
I_{\alpha, \beta}=\frac{1}{\varphi^{+}(q)} \sum_{\chi(\bmod q)}^{+} L\left(\frac{1}{2}+\alpha, \chi\right) L\left(\frac{1}{2}+\beta, \bar{\chi}\right)|A(\chi)|^{2},
$$

where

$$
A(\chi)=\sum_{a \leq q^{\kappa}} \frac{\boldsymbol{\alpha}_{a} \chi(a)}{\sqrt{a}},
$$

and $\boldsymbol{\alpha}_{a}$ is an arbitrary sequence of complex numbers satisfying $\boldsymbol{\alpha}_{a} \ll_{\varepsilon} a^{\varepsilon}$.

For technical convenience we assume that $q$ is prime throughout the paper. Note that in this case, the number of primitive characters is $\varphi^{*}(q)=q-2$, and we have $\varphi^{+}(q)=(q-3) / 2$. It is likely that our methods could be adapted to the case of general $q$ with more effort.

The following is our main theorem.

Theorem 1.1. Suppose that $q$ is prime. Let $\alpha, \beta \in \mathbb{C}$ satisfy

$$
|\operatorname{Re}(\alpha)|,|\operatorname{Re}(\beta)| \ll(\log q)^{-1} \quad \text { and } \quad|\operatorname{Im}(\alpha)|,|\operatorname{Im}(\beta)| \ll \log q .
$$

Suppose that $\kappa<1 / 2+1 / 202$. Then

$$
\begin{aligned}
I_{\alpha, \beta}=\zeta(1+\alpha+\beta) & \sum_{\substack{d a, d b \leq q^{\kappa} \\
(a, b)=1}} \frac{\boldsymbol{\alpha}_{d a} \overline{\boldsymbol{\alpha}_{d b}}}{d a^{1+\beta} b^{1+\alpha}} \\
& +\left(\frac{q}{\pi}\right)^{-(\alpha+\beta)} \frac{\Gamma\left(\frac{1 / 2-\alpha}{2}\right) \Gamma\left(\frac{1 / 2-\beta}{2}\right)}{\Gamma\left(\frac{1 / 2+\alpha}{2}\right) \Gamma\left(\frac{1 / 2+\beta}{2}\right)} \zeta(1-\alpha-\beta) \sum_{\substack{d a, d b \leq q^{\kappa} \\
(a, b)=1}} \frac{\boldsymbol{\alpha}_{d a} \overline{\boldsymbol{\alpha}_{d b}}}{d a^{1-\alpha} b^{1-\beta}}+O\left(q^{-\delta_{0}}\right)
\end{aligned}
$$

for some $\delta_{0}>0$.

The range of $\kappa$ can be enlarged if we know more about the Dirichlet polynomial $A(\chi)$. Let $\gamma$ be a smooth function supported in [1,2] such that $\gamma^{(j)} \ll_{j} q^{\varepsilon}$ for any fixed $j \geq 0$. Suppose that $\boldsymbol{\alpha}=\boldsymbol{\eta} * \boldsymbol{\lambda}$, where $\boldsymbol{\eta}_{a_{1}}, \boldsymbol{\lambda}_{a_{2}}$ are two sequences of complex numbers supported on [1, $\left.A_{1}\right]$ and $\left[1, A_{2}\right]$, respectively, with $A_{1}=q^{\kappa_{1}}, A_{2}=q^{\kappa_{2}}$ and $\kappa=\kappa_{1}+\kappa_{2}$, and satisfy $\boldsymbol{\eta}_{a}, \boldsymbol{\lambda}_{a} \ll a^{\varepsilon}$. Friedlander and Iwaniec [7] showed that

$$
\frac{1}{\varphi^{*}(q)} \sum_{\chi(\bmod q)}^{*}\left|\sum_{n} \frac{\chi(n)}{\sqrt{n}} \gamma\left(\frac{n}{N}\right)\right|^{2}|A(\chi)|^{2} \ll_{\varepsilon} q^{\varepsilon}+q^{-3 / 4+5 \kappa / 4+\varepsilon}\left(A_{1}+A_{2}\right)^{1 / 4},
$$

if $N \ll q^{1 / 2+\varepsilon}$. When $A_{1} \asymp A_{2}$, their result gives an upper bound of $O_{\varepsilon}\left(q^{\varepsilon}\right)$ provided that $\kappa<1 / 2+1 / 22$.

\footnotetext{
${ }^{1}$ See Lemma 6.2
} 
Theorem 1.2. Assume as above and suppose further that $9 \kappa+\max \left\{\kappa_{1}, \kappa_{2}\right\}<5$. Then (1.2) holds for some $\delta_{0}>0$.

If $\kappa_{1}=\kappa_{2}$, then Theorem 1.2 allows us to take $\kappa<1 / 2+1 / 38$.

Another case of special interest is when $\boldsymbol{\alpha}=\boldsymbol{\eta} * \boldsymbol{\lambda}$ with $\boldsymbol{\eta}_{a}$ being smooth coefficients up to $q^{1 / 2+\varepsilon}$ and $\boldsymbol{\lambda}_{a}$ being arbitrary and as long as possible. This can be viewed as an analogue of Hough's result [12; Theorem 4] (see also [23; Theorem 1.1]), which gives an asymptotic formula for the fourth moment of Dirichlet $L$-functions twisted by the square of a Dirichlet polynomial of length less than $q^{1 / 32}$.

Suppose that

$$
\boldsymbol{\eta}_{a_{1}}=\eta\left(\frac{a_{1}}{A_{1}}\right)
$$

where $\eta$ is a smooth function supported in $[1,2]$ such that $\eta^{(j)} \ll_{j} q^{\varepsilon}$ for any fixed $j \geq 0$. If $N, A_{1} \ll q^{1 / 2+\varepsilon}$, then Watt $[\mathbf{2 1}]$ proved that

$$
\frac{1}{\varphi^{*}(q)} \sum_{\chi(\bmod q)}^{*}\left|\sum_{n} \frac{\chi(n)}{\sqrt{n}} \gamma\left(\frac{n}{N}\right)\right|^{2}|A(\chi)|^{2} \ll_{\varepsilon} q^{\varepsilon}+q^{\vartheta-1 / 2+\varepsilon} A_{2}^{2},
$$

where $\vartheta=7 / 64$. This yields an upper bound of $O_{\varepsilon}\left(q^{\varepsilon}\right)$ provided that $\kappa_{2}<1 / 4-\vartheta / 2$.

Theorem 1.3. Assume as above and suppose further that $\kappa_{2}<1 / 14-\vartheta / 7$ with $\vartheta=$ $7 / 64$. Then (1.2) holds for some $\delta_{0}>0$.

As an application of Theorem 1.1, we obtain the order of magnitude of the third moment of Dirichlet $L$-functions.

Theorem 1.4. Suppose that $q$ is prime. Then

$$
\frac{1}{\varphi^{*}(q)} \sum_{\chi(\bmod q)}^{*}\left|L\left(\frac{1}{2}, \chi\right)\right|^{3} \asymp(\log q)^{9 / 4} .
$$

We remark that it is also possible to obtain upper bounds on all moments below the fourth by adapting the work of Radziwiłł and Soundararajan [18] and Hough [12] (see also $[\mathbf{9}])$.

An important application of the twisted second moment of Dirichlet $L$-functions is in regard to non-vanishing of Dirichlet $L$-functions at the central point $s=1 / 2$. It is widely believed that $L(1 / 2, \chi) \neq 0$ for all primitive characters $\chi$. At least $34 \%$ of Dirichlet $L$ functions in the family of primitive characters, to a large modulus $q$, are known to not vanish at the central point [3] (see also [14]). Our results here, together with the mollifier method, may be used to give a slight improvement of such a result. However, we note that in the case of large prime $q$, Khan and Ngo [15], using the "twisted" mollifier introduced by [20] and [16], have obtained a non-vanishing proportion of 3/8. Improving that using the usual mollifier would require a mollifier with $\kappa>3 / 5$, which seems out of reach of the current techniques.

The use of Theorem 2 of [6] and our Proposition 3.2 below also suffices to break the $\frac{1}{2}$-barrier. With those results, Theorem 1.1 holds provided that $\kappa<1 / 2+1 / 526$.

1.1. Comparison with the $t$-aspect analogue. We close the introduction with a brief discussion of the main differences between our work and the work of Bettin, Chandee and Radziwill's on the twisted second moment of the Riemann zeta-function $[2]$. 
In [2], after applying the approximate functional equation and dyadic decompositions, the main object to study is of the form

$$
\frac{1}{\sqrt{A B M N}} \sum_{\substack{a \asymp A, b \asymp B \\ m \asymp M, n \asymp N, M N \ll T^{1+\varepsilon}}} \sum_{\substack{a \\ \boldsymbol{\beta}_{b}}} \sum_{W}\left(T \log \frac{a m}{b n}\right),
$$

where $W$, say, is some compactly supported smooth function satisfying $W^{(j)} \ll_{j} T^{\varepsilon}$ for any fixed $j \geq 0$. The diagonal contribution $a m=b n$ may be extracted and is fairly easy to understand. For the remaining terms, write $a m-b n=r$ with $r \neq 0$. The rapid decay of the Fourier transform implies that the contribution of the terms with $|r|>T^{-1+\varepsilon} \sqrt{A B M N}$ is negligible. The off-diagonal contribution is then essentially

$$
\frac{1}{\sqrt{A B M N}} \sum_{0<|r| \leq T^{-1+\varepsilon} \sqrt{A B M N}} \sum \sum_{\substack{a m-b b=r \\ a \asymp A, b \asymp B \\ m \asymp M, n \asymp N, M N \ll T^{1+\varepsilon}}} \sum_{\substack{a \\ m}} \boldsymbol{\alpha}_{a} .
$$

In particular, this is null unless $A M \asymp B N$. Writing $a m-b n=r$ as a congruence condition modulo $b$ and applying the Poisson summation formula transform the above expression to an exponential sum roughly of the form

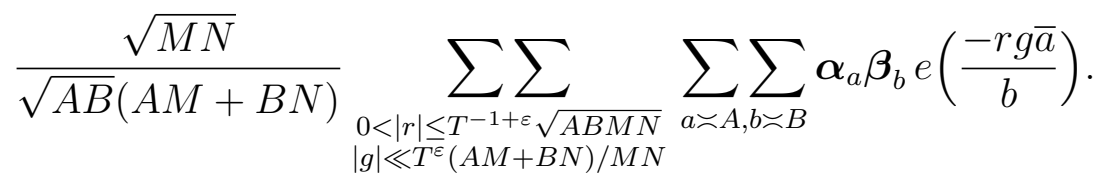

Trivially, this is bounded by $T^{-1+\varepsilon} A B \ll T^{2 \kappa-1+\varepsilon}$. So any extra power saving is sufficient to break the $\frac{1}{2}$-barrier. Bettin, Chandee and Radziwiłł [2] gained this by utilizing an estimate for sums of Kloosterman fractions in [1] (or, [6]).

In our situation, we use the approximate functional equation and apply character orthogonality, as well as dyadic decompositions, to reduce the problem to understanding sums of the form

$$
\frac{1}{\sqrt{A B M N}} \sum_{\substack{a m \equiv \pm b n\left(\bmod \\ a \asymp A, b \preceq B \\ m \asymp M, n \asymp N, M N \ll q^{1+\varepsilon}\right.}} \sum_{\substack{a \\ m \asymp M}} \boldsymbol{\alpha}_{b} .
$$

Diagonal main terms arise from $a m=b n$. We set this contribution aside, and study the remaining terms. At this point, we can, as above, write the congruence condition modulo $q$ as $a m \mp b n=q r$ with $r \neq 0$, and switch to a congruence condition modulo $b$. The Poisson summation formula then leads to an exponential sum roughly of the form

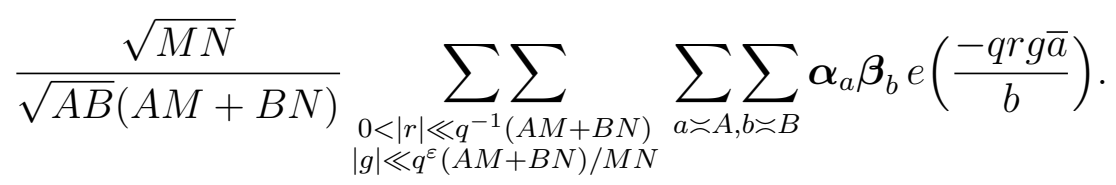

The results on cancellation in sums of Kloosterman fractions $[\mathbf{6}, \mathbf{1}]$ are still applicable, but only work in the "balanced" case when $A M$ and $B N$ are more or less of the same size. Roughly speaking, the $t$-aspect averaging yields the constraint $A M \asymp B N$ and ensures that $r, g \ll T^{\kappa-1 / 2+\varepsilon}$ in (1.4). In our situation (1.5), we lack the condition $A M \asymp B N$, and therefore the ranges of summation of $r$ and $g$ can be as large as $q^{\kappa+\varepsilon}$. The trivial bound, which can be $O_{\varepsilon}\left(q^{2 \kappa-1 / 2+\varepsilon}\right)$, is worse in our case as well. So a different method is required for the "unbalanced" regime when $A M$ and $B N$ are of 
rather different sizes. Furthermore, it is impossible to ignore the contribution of these terms. This contribution is genuinely large, and, as it turns out, will cancel out with the contribution from the principal character modulo $q$. A similar phenomenon already arose in Young's work on the fourth moment of Dirichlet $L$-functions [22]. We remark that it is possible to show that $I_{\alpha, \beta} \ll_{\varepsilon} q^{\varepsilon}$ for some $\kappa>1 / 2$ without considering the unbalanced case [5].

We begin the treatment of the unbalanced regime by applying the Poisson summation formula to introduce exponential phases. The zero frequency cancels out with the contribution from the principal character modulo $q$. We bound the contribution of the non-zero frequencies with a delicate argument involving the additive large sieve inequality. The phases of the exponentials are rational fractions, and we divide these fractions into two classes: "good" fractions and "bad" fractions. The good fractions are far apart from each other, and we can immediately apply the additive large sieve inequality to get a saving. The bad fractions can be close together, which weakens the large sieve inequality, but we still obtain a saving since there are comparatively few of these bad fractions.

Lastly, we remark that with our two different methods for the two different regimes, the critical ranges of summation in (1.5) are when $A \asymp B \asymp N \asymp q^{\kappa}$ and $M \asymp q^{2-3 \kappa}$. This explains why the ranges of $\kappa$ for the asymptotic formula in our theorems are slightly smaller than the corresponding ranges for the upper bound.

Remark 1.5. Throughout the paper $\varepsilon$ denotes an arbitrarily small positive number whose value may change from one line to the next.

\section{INITIAL MANIPULATIONS}

We start by recalling the orthogonality property of characters and the approximate functional equation.

Lemma 2.1 (Orthogonality). For $(m n, q)=1$ we have

$$
\sum_{\chi(\bmod q)}^{+} \chi(m) \bar{\chi}(n)=\frac{1}{2} \sum_{\substack{d|q \\ d|(m \pm n)}} \mu\left(\frac{q}{d}\right) \varphi(d)=\frac{1}{2} \sum_{q \mid(m \pm n)} \varphi(q)-1 .
$$

Proof. The proof is standard. See, for example, [14; (3.1) and (3.2)].

Lemma 2.2 (Approximate functional equation). Let $\chi$ be an even primitive character and let $G(s)$ be an even entire function of rapid decay in any fixed strip $|\operatorname{Re}(s)| \leq C$ satisfying $G(0)=1$. Let

$$
X_{ \pm}(s)=G(s) \frac{\Gamma\left(\frac{1 / 2 \pm \alpha+s}{2}\right) \Gamma\left(\frac{1 / 2 \pm \beta+s}{2}\right)}{\Gamma\left(\frac{1 / 2+\alpha}{2}\right) \Gamma\left(\frac{1 / 2+\beta}{2}\right)}
$$

and

$$
V_{ \pm}(x)=\frac{1}{2 \pi i} \int_{(\varepsilon)} X_{ \pm}(s) x^{-s} \frac{d s}{s}
$$

Then

$$
L\left(\frac{1}{2}+\alpha, \chi\right) L\left(\frac{1}{2}+\beta, \bar{\chi}\right)=\sum_{m, n \geq 1} \frac{\chi(m) \bar{\chi}(n)}{m^{1 / 2+\alpha} n^{1 / 2+\beta}} V_{+}\left(\frac{\pi m n}{q}\right)
$$




$$
+\left(\frac{q}{\pi}\right)^{-(\alpha+\beta)} \sum_{m, n \geq 1} \frac{\chi(m) \bar{\chi}(n)}{m^{1 / 2-\beta} n^{1 / 2-\alpha}} V_{-}\left(\frac{\pi m n}{q}\right) .
$$

Proof. The proof is standard and can be easily derived following Theorem 5.3 of $[\mathbf{1 3}]$.

\section{Remark 2.3.}

- It is convenient to prescribe certain conditions on the function $G$. To be precise, we assume $G(s)$ is invariant under the transformations $\alpha \rightarrow-\beta, \beta \rightarrow-\alpha$, and vanishes at $s= \pm(\alpha+\beta) / 2$. An admissible choice of $G$ is

$$
G(s)=\frac{\left(\frac{\alpha+\beta}{2}\right)^{2}-s^{2}}{\left(\frac{\alpha+\beta}{2}\right)^{2}} e^{s^{2}},
$$

but there is no need to specify a particular function $G$.

- If $|\operatorname{Re}(\alpha)|,|\operatorname{Re}(\beta)| \ll(\log q)^{-1}$ and $|\operatorname{Im}(\alpha)|,|\operatorname{Im}(\beta)| \leq T$, then Stirling's approximation gives

$$
x^{j} V_{ \pm}^{(j)}(x) \ll_{j, C}(1+|x| / T)^{-C}
$$

for any fixed $j \geq 0$ and $C>0$.

From Lemma 2.2 and Lemma 2.1 we get

$$
\begin{aligned}
I_{\alpha, \beta}= & \sum_{\substack{a, b \leq q^{\kappa} \\
m, n \geq 1}} \frac{\boldsymbol{\alpha}_{a} \overline{\boldsymbol{\alpha}_{b}}}{\sqrt{a b} m^{1 / 2+\alpha} n^{1 / 2+\beta}} V_{+}\left(\frac{\pi m n}{q}\right) \frac{1}{\varphi^{+}(q)} \sum_{\chi(\bmod q)}^{+} \chi(a m) \bar{\chi}(b n) \\
& +\left(\frac{q}{\pi}\right)^{-(\alpha+\beta)} \sum_{\substack{a, b \leq q^{\kappa} \\
m, n \geq 1}} \frac{\boldsymbol{\alpha}_{a} \overline{\boldsymbol{\alpha}_{b}}}{\sqrt{a b} m^{1 / 2-\beta} n^{1 / 2-\alpha}} V_{-}\left(\frac{\pi m n}{q}\right) \frac{1}{\varphi^{+}(q)} \sum_{\chi(\bmod q)}^{+} \chi(a m) \bar{\chi}(b n) \\
= & J_{\alpha, \beta}^{+}+\left(\frac{q}{\pi}\right)^{-(\alpha+\beta)} J_{-\beta,-\alpha}^{-},
\end{aligned}
$$

where

$$
\begin{aligned}
& J_{\alpha, \beta}^{+}=\frac{\varphi(q)}{2 \varphi^{+}(q)} \sum_{\substack{a, b \leq q^{\kappa} \\
a m \equiv \pm b n(\bmod q) \\
(m n, q)=1}} \frac{\boldsymbol{\alpha}_{a} \overline{\boldsymbol{\alpha}_{b}}}{\sqrt{a b} m^{1 / 2+\alpha} n^{1 / 2+\beta}} V_{+}\left(\frac{\pi m n}{q}\right) \\
&-\frac{1}{\varphi^{+}(q)} \sum_{\substack{a, b \leq q^{\kappa} \\
(m n, q)=1}} \frac{\boldsymbol{\alpha}_{a} \overline{\boldsymbol{\alpha}_{b}}}{\sqrt{a b m^{1 / 2+\alpha}} n^{1 / 2+\beta}} V_{+}\left(\frac{\pi m n}{q}\right),
\end{aligned}
$$

and $J^{-}$is the same but with $V_{-}$in place of $V_{+}$. Note from Remark 2.3 that the condition $(m n, q)=1$ may be omitted with the cost of an error of size $O_{\varepsilon}\left(q^{\kappa-3 / 2+\varepsilon}\right)$. The same error applies when we replace $\varphi(q)$ by $q$ and $\varphi^{+}(q)$ by $q / 2$. So, up to an error term of size $O_{\varepsilon}\left(q^{\kappa-3 / 2+\varepsilon}\right)$, we have

$$
\begin{aligned}
J_{\alpha, \beta}^{+} & =\sum_{\substack{a, b \leq q^{\kappa} \\
a m \equiv \pm b n(\bmod q)}} \frac{\boldsymbol{\alpha}_{a} \overline{\boldsymbol{\alpha}_{b}}}{\sqrt{a b} m^{1 / 2+\alpha} n^{1 / 2+\beta}} V_{+}\left(\frac{\pi m n}{q}\right)-\frac{2}{q} \sum_{\substack{a, b \leq q^{\kappa} \\
m, n \geq 1}} \frac{\boldsymbol{\alpha}_{a} \overline{\boldsymbol{\alpha}_{b}}}{\sqrt{a b} m^{1 / 2+\alpha} n^{1 / 2+\beta}} V_{+}\left(\frac{\pi m n}{q}\right) \\
= & \mathcal{M}_{\alpha, \beta}^{+}+S_{\alpha, \beta}^{+}-\frac{2}{q} \sum_{\substack{a, b \leq q^{\kappa} \\
m, n \geq 1}} \frac{\boldsymbol{\alpha}_{a} \overline{\boldsymbol{\alpha}_{b}}}{\sqrt{a b} m^{1 / 2+\alpha} n^{1 / 2+\beta}} V_{+}\left(\frac{\pi m n}{q}\right),
\end{aligned}
$$


where $\mathcal{M}_{\alpha, \beta}^{+}$and $S_{\alpha, \beta}^{+}$are the contributions from the diagonal terms $a m=b n$ and the off-diagonal terms $a m \neq b n$ in the first sum, respectively. We similarly define $\mathcal{M}_{-\beta,-\alpha}^{-}$ and $S_{-\beta,-\alpha}^{-}$.

Much of this paper is spent studying the off-diagonal terms $S^{ \pm}$. We shall complete the proofs of Theorem 1.1 and Theorems 1.2, 1.3 in Sections 4 and 5. We just finish this section with a partial evaluation of $\mathcal{M}^{ \pm}$. Replacing $a, b$ by $d a, d b$ with $(a, b)=1$, we see that $m=b n^{\prime}$ and $n=a n^{\prime}$ for some $n^{\prime} \in \mathbb{N}$. Hence

$$
\begin{aligned}
\mathcal{M}_{\alpha, \beta}^{+} & =\sum_{\substack{d a, d b \leq q^{\kappa} \\
(a, b)=1}} \frac{\boldsymbol{\alpha}_{d a} \overline{\boldsymbol{\alpha}_{d b}}}{d a^{1+\beta} b^{1+\alpha}} \sum_{n \geq 1} \frac{1}{n^{1+\alpha+\beta}} V_{+}\left(\frac{\pi a b n^{2}}{q}\right) \\
& =\sum_{\substack{d a, d b \leq q^{\kappa} \\
(a, b)=1}} \frac{\boldsymbol{\alpha}_{d a} \overline{\boldsymbol{\alpha}_{d b}}}{d a^{1+\beta} b^{1+\alpha}} \frac{1}{2 \pi i} \int_{(\varepsilon)} X_{+}(s)\left(\frac{q}{\pi a b}\right)^{s} \zeta(1+\alpha+\beta+2 s) \frac{d s}{s},
\end{aligned}
$$

and a similar expression holds for $\mathcal{M}^{-}$.

\section{MAIN PROpOSITIONS}

In this section we focus on the sum

$$
\sum_{\substack{a m \equiv \pm b n(\bmod q) \\ a m \neq b n}} \boldsymbol{\alpha}_{a} \boldsymbol{\beta}_{b}
$$

over dyadic intervals. Theorem 1.1 is deduced from the following two propositions. The first result essentially treats the case when $m$ and $n$ are close, while the second one deals with the case when $m$ and $n$ are far apart.

Proposition 3.1. Let $A, B, M, N \geq 1$, and let $\boldsymbol{\alpha}_{a}, \boldsymbol{\beta}_{b}$ be two sequences of complex numbers supported on $[A, 2 A]$ and $[B, 2 B]$ satisfying $\boldsymbol{\alpha}_{a} \ll A^{\varepsilon}, \boldsymbol{\beta}_{b} \ll B^{\varepsilon}$. Let $W_{1}$ and $W_{2}$ be smooth functions supported in $[1,2]$ such that $W_{1}^{(j)}, W_{2}^{(j)} \ll_{j} q^{\varepsilon}$ for any fixed $j \geq 0$. Let

$$
\mathcal{S}=\frac{1}{\sqrt{A B M N}} \sum_{\substack{a m \equiv \pm b n(\bmod q) \\ a m \neq b n}} \boldsymbol{\alpha}_{a} \boldsymbol{\beta}_{b} W_{1}\left(\frac{m}{M}\right) W_{2}\left(\frac{n}{N}\right) .
$$

Then

$$
\mathcal{S} \ll_{\varepsilon} q^{-1+\varepsilon} \sqrt{A B M N}
$$

If $A B M N \gg q^{2-\varepsilon}$, then

$$
\mathcal{S}=\mathcal{M}_{1}^{+}+\mathcal{M}_{1}^{-}+\mathcal{E}
$$

where

$$
\mathcal{M}_{1}^{ \pm}=\frac{1}{\sqrt{A B M N}} \sum_{\substack{d \geq 1 \\ r \neq 0}} \sum_{(a, b)=1} \boldsymbol{\alpha}_{d a} \boldsymbol{\beta}_{d b} \int W_{1}\left(\frac{b x}{M}\right) W_{2}\left(\frac{ \pm(a b x-q r)}{b N}\right) d x
$$

and

$$
\begin{gathered}
\mathcal{E} \ll_{\varepsilon} q^{-17 / 20+\varepsilon}(A B)^{-3 / 20}(A M+B N)^{17 / 10}(A+B)^{1 / 4}(M N)^{-17 / 20} \\
+q^{-1+\varepsilon}(A B)^{-1 / 8}(A M+B N)^{2}(A+B)^{1 / 8}(M N)^{-1}
\end{gathered}
$$


We remark that the main term is symmetric by the change of variables $\pm a b y=$ $a b x-q r$. The error term is similarly symmetric upon switching $A$ for $B$ and $M$ for $N$, so we may assume without loss of generality that $A M \ll B N$, say.

Proof. To prove (3.1), we write $a m \mp b n=q r$. Then

$$
0<|r| \leq R=4(A M+B N) q^{-1}
$$

and

$$
\mathcal{S}=\frac{1}{\sqrt{A B M N}} \sum_{0<|r| \leq R} \sum_{a m \mp b n=q r} \boldsymbol{\alpha}_{a} \boldsymbol{\beta}_{b} W_{1}\left(\frac{m}{M}\right) W_{2}\left(\frac{n}{N}\right) .
$$

We can take the innermost sum over all $a$ and $m$, and the sums over $b$ and $n$ being over all divisors of $(a m-q r)$. So, by symmetry,

$$
\mathcal{S} \ll_{\varepsilon} \frac{q^{\varepsilon} R}{\sqrt{A B M N}} \min \{A M, B N\} \ll_{\varepsilon} q^{-1+\varepsilon} \sqrt{A B M N},
$$

and we obtain (3.1).

We next prove (3.2). We proceed from (3.5). Without loss of generality, assume that $A M \ll B N$ (consequently, we also have $q R \ll B N)$. This simplifies the decision on which variable to eliminate. We will eliminate $n$ in this case, so we first introduce Mellin inversion to write

$$
\begin{aligned}
W_{2}\left(\frac{n}{N}\right) & =W_{2}\left(\frac{ \pm(a m-q r)}{b N}\right) \\
& =\frac{1}{(2 \pi i)^{4}} \int_{(\varepsilon)} \int_{(\varepsilon)} \int_{(\varepsilon)} \int_{(\varepsilon)} \widehat{f_{ \pm}}(u, v, w, z) a^{-u} b^{-v} m^{-w} r^{-z} d u d v d w d z .
\end{aligned}
$$

Note that by integration by parts $j$ times on each variable of the expression

$$
\widehat{f_{ \pm}}(u, v, w, z)=\int_{0}^{\infty} \int_{0}^{\infty} \int_{0}^{\infty} \int_{0}^{\infty} a^{u-1} b^{v-1} m^{w-1} r^{z-1} W_{2}\left(\frac{ \pm(a m-q r)}{b N}\right) d a d b d m d r
$$

we have

$\widehat{f_{ \pm}}(u, v, w, z) \ll_{\varepsilon, j} A^{\operatorname{Re}(u)} B^{\operatorname{Re}(v)} M^{\operatorname{Re}(w)} R^{\operatorname{Re}(z)}\left(q^{-\varepsilon}(1+|u|)(1+|v|)(1+|w|)(1+|z|)\right)^{-j}$

uniformly for $\operatorname{Re}(u), \operatorname{Re}(v), \operatorname{Re}(w), \operatorname{Re}(z) \geq \varepsilon$, and for any fixed $j \geq 0$. Hence we may restrict the $u, v, w, z$-integrals to

$$
|u|,|v|,|w|,|z| \ll q^{\varepsilon} .
$$

Let $d=(a, b)$ (note that this implies $d \mid r)$. We can now remove $n$ by writing $a m \mp b n=$ $q r$ as $m \equiv q(r / d) \overline{a / d}(\bmod b / d)$. The Poisson summation formula then yields

$$
\begin{aligned}
\sum_{\substack{m, n \\
a m \mp b n=q r}} m^{-w} W_{1}\left(\frac{m}{M}\right) & =\sum_{m \equiv q(r / d) \overline{a / d}(\bmod b / d)} m^{-w} W_{1}\left(\frac{m}{M}\right) \\
& =\sum_{g \in \mathbb{Z}} e\left(\frac{-q(r / d) g \overline{a / d}}{b / d}\right) \int\left(\frac{b x}{d}\right)^{-w} W_{1}\left(\frac{b x}{d M}\right) e(g x) d x .(3.7
\end{aligned}
$$

Note that the integral is over $x \asymp d M / B \asymp d M N /(A M+B N)$. 
For the term $g=0$, we fold back the Mellin inversion and get the terms $\mathcal{M}_{1}^{ \pm}$in (3.3). The restriction $d|r| \leq R$ may be removed due to the support of $W$. For the terms $g \neq 0$, integration by parts $j$ times implies that

$$
\int\left(\frac{b x}{d}\right)^{-w} W_{1}\left(\frac{b x}{d M}\right) e(g x) d x \ll_{j} \frac{d M^{1-\operatorname{Re}(w)}}{B}\left(\frac{(1+|w|) B}{g d M}\right)^{j}
$$

for any fixed $j \geq 0$. So we may restrict the sum in (3.7) to $0<|g| \leq G / d$, where

$$
G=\frac{q^{\varepsilon} B}{M} \asymp \frac{q^{\varepsilon}(A M+B N)}{M N} .
$$

Hence,

$$
\mathcal{S}=\mathcal{M}_{1}^{+}+\mathcal{M}_{1}^{-}+\mathcal{E}+O_{C}\left(q^{-C}\right)
$$

with

$$
\begin{aligned}
& \mathcal{E} \ll \frac{1}{\sqrt{A B M N}} \sum_{d \leq R} \int_{(\varepsilon)} \int_{(\varepsilon)} \int_{(\varepsilon)} \int_{(\varepsilon)} \int_{x \asymp d M N /(A M+B N)} \\
& x^{-\operatorname{Re}(w)}\left|\widehat{f_{ \pm}}(u, v, w, z)\right|\left|Z_{d}(x)\right| d x d u d v d w d z,
\end{aligned}
$$

where

$$
Z_{d}(x)=d^{-(u+v+z)} \sum_{\substack{0<|r| \leq R / d \\ 0<|g| \leq G / d}} \sum_{(a, b)=1} a^{-u} b^{-(v+w)} r^{-z} \boldsymbol{\alpha}_{d a} \boldsymbol{\beta}_{d b} e\left(\frac{-q r g \bar{a}}{b}+g x\right) W_{1}\left(\frac{b x}{M}\right) .
$$

The above expression is in the form of Theorem 1 of $[\mathbf{1}]$. We apply this and get

$$
\begin{aligned}
Z_{d}(x) \ll_{\varepsilon} q^{\varepsilon} & \left(\frac{A B R G}{d^{4}}\right)^{1 / 2}\left(1+\frac{q R G}{A B}\right)^{1 / 2} \\
& \left(\left(\frac{A B R G}{d^{4}}\right)^{7 / 20}\left(\frac{A}{d}+\frac{B}{d}\right)^{1 / 4}+\left(\frac{A B}{d^{2}}\right)^{3 / 8}\left(\frac{R G}{d^{2}}\right)^{1 / 2}\left(\frac{A}{d}+\frac{B}{d}\right)^{1 / 8}\right) \\
\ll & \ll_{\varepsilon} d^{-2} q^{-1 / 2+\varepsilon} \frac{(A M+B N)^{2}}{M N} \\
& \left(\frac{(A B)^{7 / 20}(A M+B N)^{7 / 10}(A+B)^{1 / 4}}{d^{33 / 20}(q M N)^{7 / 20}}+\frac{(A B)^{3 / 8}(A M+B N)(A+B)^{1 / 8}}{d^{15 / 8}(q M N)^{1 / 2}}\right) .
\end{aligned}
$$

Plugging this into (3.9) and using the bound in (3.6) we obtain (3.4).

Proposition 3.2. Assume the conditions of Proposition 3.1 and that $B M \ll q^{1-\varepsilon}$, $A \ll N, B \gg M$. Then

$$
\mathcal{S}=\mathcal{M}_{2}+\mathcal{E}
$$

where

$$
\mathcal{M}_{2}=\frac{2}{\sqrt{A B M N}} \sum_{a, b} \sum_{m} \boldsymbol{\alpha}_{a} \boldsymbol{\beta}_{b} W_{1}\left(\frac{m}{M}\right) \int W_{2}\left(\frac{q x}{N}\right) d x
$$

and

$$
\mathcal{E} \ll_{\varepsilon}\left(\frac{q}{B M}\right)^{-1 / 6+\varepsilon}+q^{\varepsilon}\left(\sqrt{\frac{A}{N}}+\sqrt{\frac{M}{B}}\right) .
$$


Proof. Applying the Poisson summation formula over $n$ gives

$$
\begin{aligned}
\mathcal{S} & =\frac{1}{\sqrt{A B M N}} \sum_{a, b} \sum_{m} \boldsymbol{\alpha}_{a} \boldsymbol{\beta}_{b} W_{1}\left(\frac{m}{M}\right) \sum_{n \equiv \pm a m \bar{b}(\bmod q)} W_{2}\left(\frac{n}{N}\right) \\
& =\frac{\sqrt{N}}{q \sqrt{A B M}} \sum_{a, b} \sum_{m} \sum_{h \in \mathbb{Z}} \boldsymbol{\alpha}_{a} \boldsymbol{\beta}_{b} e\left(\frac{ \pm a h m \bar{b}}{q}\right) W_{1}\left(\frac{m}{M}\right) \widehat{W}_{2}\left(\frac{h N}{q}\right) .
\end{aligned}
$$

The contribution of the term $h=0$ corresponds to the term $\mathcal{M}_{2}$ in (3.10). For $h \neq 0$ the rapid decay of the Fourier transform means we may restrict the sum over $h$ to $0<|h| \leq H$, where

Thus we have

$$
H=\frac{q^{1+\varepsilon}}{N}
$$

$$
\mathcal{S}=\mathcal{M}_{2}+\mathcal{E}+O_{C}\left(q^{-C}\right)
$$

where

$$
\begin{aligned}
\mathcal{E} & =\frac{\sqrt{N}}{q \sqrt{A B M}} \sum_{a, b} \sum_{m} \sum_{0<|h| \leq H} \boldsymbol{\alpha}_{a} \boldsymbol{\beta}_{b} e\left(\frac{ \pm a h m \bar{b}}{q}\right) W_{1}\left(\frac{m}{M}\right) \widehat{W}_{2}\left(\frac{h N}{q}\right) \\
& =\frac{\sqrt{N}}{q \sqrt{A B M}} \sum_{l} \sum_{u \in \mathcal{U}_{l}} \nu_{l}(u) \sum_{a} \sum_{0<|h| \leq H} \boldsymbol{\alpha}_{a} e\left(\frac{ \pm a h u}{q}\right) \widehat{W}_{2}\left(\frac{h N}{q}\right) .
\end{aligned}
$$

Here

$$
\mathcal{U}_{l}=\{0<u<q: \exists B / l \leq b \leq 2 B / l, M / l \leq m \leq 2 M / l,(b, m)=1, m \bar{b} \equiv u(\bmod q)\}
$$

and

$$
\nu_{l}(u)=\sum_{\substack{m \bar{b} \equiv u(\bmod q) \\(b, m)=1}} \boldsymbol{\beta}_{l b} W_{1}\left(\frac{l m}{M}\right)
$$

Note that if

$$
\left(b_{1}, m_{1}\right)=\left(b_{2}, m_{2}\right)=1 \quad \text { and } \quad m_{1} \overline{b_{1}} \equiv m_{2} \overline{b_{2}} \equiv u(\bmod q),
$$

then $b_{1} m_{2} \equiv b_{2} m_{1}(\bmod q)$. Since $B M \ll q^{1-\varepsilon}$, it follows that $b_{1} m_{2}=b_{2} m_{1}$, and hence $b_{1}=b_{2}$ and $m_{1}=m_{2}$. So given $u \in \mathcal{U}_{l}$, the existence of the pair $(b, m)$ in $(3.12)$ is unique. In particular we get

$$
\# \mathcal{U}_{l} \ll \frac{B M}{l^{2}} \quad \text { and } \quad \nu_{l}(u) \ll_{\varepsilon} q^{\varepsilon} .
$$

Given $0<X<q$, we call a pair $\left(u_{1}, u_{2}\right)$ " $(l, X)$-bad" if $u_{1}, u_{2} \in \mathcal{U}_{l}$ and

$$
u_{1}-u_{2} \equiv s(\bmod q)
$$

with $0<|s| \leq X$. Define $\mathcal{U}_{l}^{\text {bad }} \subset \mathcal{U}_{l}$ to be the set consisting of all $u$ 's which belong to at least one such pair, and set $\mathcal{U}_{l}^{\text {good }}=\mathcal{U}_{l} \backslash \mathcal{U}_{l}^{\text {bad }}$.

Let

$$
\begin{aligned}
Y_{l}=\#\left\{B / l \leq b_{1}, b_{2} \leq 2 B / l, M / l \leq m_{1}, m_{2} \leq 2 M / l, 0<|s| \leq X:\right. \\
\left.\left(b_{1}, m_{1}\right)=\left(b_{2}, m_{2}\right)=1 \text { and } m_{1} \overline{b_{1}}-m_{2} \overline{b_{2}} \equiv s(\bmod q)\right\} .
\end{aligned}
$$


We have

$$
\begin{aligned}
m_{1} \overline{b_{1}}-m_{2} \overline{b_{2}} \equiv s(\bmod q) & \Longleftrightarrow s b_{1} b_{2}+b_{1} m_{2}-b_{2} m_{1} \equiv 0(\bmod q) \\
& \Longleftrightarrow\left(s b_{1}-m_{1}\right)\left(s b_{2}+m_{2}\right) \equiv-m_{1} m_{2}(\bmod q) .
\end{aligned}
$$

The facts that given $m_{1}, m_{2}$ and the product $\left(s b_{1}-m_{1}\right)\left(s b_{2}+m_{2}\right) \neq 0$, the number of triples $\left(b_{1}, b_{2}, s\right)$ is $\ll_{\varepsilon} q^{\varepsilon}$, and that $\left(s b_{1}-m_{1}\right)\left(s b_{2}+m_{2}\right) \ll X^{2}(B / l)^{2}$ lead to

$$
Y_{l} \ll_{\varepsilon} q^{\varepsilon}\left(\frac{M}{l}\right)^{2}\left(1+\frac{X^{2} B^{2}}{l^{2} q}\right) \ll_{\varepsilon} q^{\varepsilon}\left(\frac{M^{2}}{l^{2}}+\frac{X^{2} B^{2} M^{2}}{l^{4} q}\right) .
$$

The same bound, hence, applies to $\# \mathcal{U}_{l}^{\text {bad }}$, and so

$$
\sum_{u \in \mathcal{U}_{l}^{\text {bad }}}\left|\nu_{l}(u)\right|^{2} \ll_{\varepsilon} q^{\varepsilon}\left(\frac{M^{2}}{l^{2}}+\frac{X^{2} B^{2} M^{2}}{l^{4} q}\right) .
$$

We write (3.11) as

$$
\mathcal{E}=\frac{\sqrt{N}}{q \sqrt{A B M}}\left(\sum_{l} \sum_{u \in \mathcal{U}_{l}^{\text {bad }}}+\sum_{l} \sum_{u \in \mathcal{U}_{l}^{\text {good }}}\right)=\frac{\sqrt{N}}{q \sqrt{A B M}}\left(Z_{\text {bad }}+Z_{\text {good }}\right),
$$

say. For $Z_{\text {bad }}$, we apply Cauchy's inequality, (3.14) and the additive large sieve inequality (see [17; Corollary 2.2] or [13; Theorem 7.7]), obtaining

$$
\begin{aligned}
Z_{\mathrm{bad}} & \ll \sum_{l} \sum_{u \in \mathcal{U}_{l}^{\text {bad }}}\left|\nu_{l}(u)\right|\left|\sum_{a} \sum_{0<|h| \leq H} \boldsymbol{\alpha}_{a} e\left(\frac{ \pm a h u}{q}\right) \widehat{W}_{2}\left(\frac{h N}{q}\right)\right| \\
& \ll \sum_{l}\left(\sum_{u \in \mathcal{U}_{l}^{\text {bad }}}\left|\nu_{l}(u)\right|^{2}\right)^{1 / 2}\left(\sum_{u \in \mathcal{U}_{l}^{\text {bad }}}\left|\sum_{a} \sum_{0<|h| \leq H} \boldsymbol{\alpha}_{a} e\left(\frac{ \pm a h u}{q}\right) \widehat{W}_{2}\left(\frac{h N}{q}\right)\right|^{2}\right)^{1 / 2} \\
& \ll \varepsilon q^{\varepsilon} \sum_{l}\left(\frac{M}{l}+\frac{X B M}{l^{2} q^{1 / 2}}\right)((q+A H) A H)^{1 / 2} \ll_{\varepsilon} q^{1+\varepsilon}\left(M+q^{-1 / 2} X B M\right) \sqrt{\frac{A}{N}} .
\end{aligned}
$$

For $Z_{\text {good }}$, by Cauchy's inequality and (3.13) we get

$$
\begin{aligned}
Z_{\text {good }} & \ll \sum_{l}\left(\sum_{u \in \mathcal{U}_{l}}\left|\nu_{l}(u)\right|^{2}\right)^{1 / 2}\left(\sum_{u \in \mathcal{U}_{l}^{\text {good }}}\left|\sum_{a} \sum_{0<|h| \leq H} \boldsymbol{\alpha}_{a} e\left(\frac{ \pm a h u}{q}\right) \widehat{W}_{2}\left(\frac{h N}{q}\right)\right|^{2}\right)^{1 / 2} \\
& \ll_{\varepsilon} q^{\varepsilon} \sum_{l} \frac{(B M)^{1 / 2}}{l}\left(\sum_{u \in \mathcal{U}_{l}^{\text {good }}}\left|\sum_{a} \sum_{0<|h| \leq H} \boldsymbol{\alpha}_{a} e\left(\frac{ \pm a h u}{q}\right) \widehat{W}_{2}\left(\frac{h N}{q}\right)\right|^{2}\right)^{1 / 2} .
\end{aligned}
$$

Note that for every $u_{1}, u_{2} \in \mathcal{U}_{l}^{\text {good }}$ we have $\left\|u_{1} / q-u_{2} / q\right\|>X / q$, where $\|\xi\|$ denotes the distance from $\xi \in \mathbb{R}$ to the nearest integer. Another application of the large sieve inequality yields

$$
Z_{\text {good }} \ll_{\varepsilon} q^{\varepsilon} \sum_{l} \frac{(B M)^{1 / 2}}{l}((q / X+A H) A H)^{1 / 2} \ll_{\varepsilon} q^{1+\varepsilon}\left((B M)^{1 / 2} X^{-1 / 2}+\frac{\sqrt{A B M}}{\sqrt{N}}\right) \sqrt{\frac{A}{N}} .
$$


Combining (3.15), (3.16) and choosing $X=(q / B M)^{1 / 3}$ we obtain

$$
\mathcal{E} \ll_{\varepsilon}\left(\frac{q}{B M}\right)^{-1 / 6+\varepsilon}+q^{\varepsilon}\left(\sqrt{\frac{A}{N}}+\sqrt{\frac{M}{B}}\right),
$$

and the result follows.

For Theorem 1.2 we need the following proposition instead of Proposition 3.1.

Proposition 3.3. Assume the conditions of Proposition 3.1 and that $A B M N \gg q^{2-\varepsilon}$, $A M \ll B N$. Assume also that $\boldsymbol{\alpha}=\boldsymbol{\eta} * \boldsymbol{\lambda}$, where the sequences $\boldsymbol{\eta}_{a_{1}}$ and $\boldsymbol{\lambda}_{a_{2}}$ are supported on the intervals $\left[A_{1}, 2 A_{1}\right]$ and $\left[A_{2}, 2 A_{2}\right]$ with $A=A_{1} A_{2}$, and satisfy $\boldsymbol{\eta}_{a}, \boldsymbol{\lambda}_{a} \ll a^{\varepsilon}$. Then

$$
\mathcal{S}=\mathcal{M}_{1}^{+}+\mathcal{M}_{1}^{-}+\mathcal{E}
$$

where $\mathcal{M}_{1}^{ \pm}$are defined as in (3.3) and

$$
\begin{aligned}
\mathcal{E} \ll_{\varepsilon} q^{-1+\varepsilon} & A^{1 / 4} B^{1 / 2}(A M+B N)(M N)^{-1 / 2} \\
& +q^{-3 / 4+\varepsilon} A^{1 / 4} B^{1 / 2}(A M+B N)^{1 / 2}(M N)^{-1 / 4}\left(A_{1}+A_{2}\right)^{1 / 4} .
\end{aligned}
$$

Proof. With $A B M N \gg q^{2-\varepsilon}$ and $A M \ll B N$, we recall from (3.8) that

$$
\mathcal{S}=\mathcal{M}_{1}^{+}+\mathcal{M}_{1}^{-}+\mathcal{E}+O_{C}\left(q^{-C}\right)
$$

with

$$
\begin{aligned}
& \mathcal{E} \ll \frac{1}{\sqrt{A B M N}} \sum_{d \leq R} \int_{(\varepsilon)} \int_{(\varepsilon)} \int_{(\varepsilon)} \int_{(\varepsilon)} \int_{x \asymp d M N /(A M+B N)} \\
& x^{-\operatorname{Re}(w)}\left|\widehat{f_{ \pm}}(u, v, w, z)\right|\left|Z_{d}(x)\right| d x d u d v d w d z,
\end{aligned}
$$

where

$$
Z_{d}(x)=d^{w-z} \sum_{\substack{0<|r| \leq R / d \\ 0<|g| \leq G / d}} \sum_{(a, b)=d} a^{-u} b^{-(v+w)} r^{-z} \boldsymbol{\alpha}_{a} \boldsymbol{\beta}_{b} e\left(\frac{-q r g \overline{a / d}}{b / d}+g x\right) W_{1}\left(\frac{b x}{d M}\right) .
$$

Using the fact that $\boldsymbol{\alpha}=\boldsymbol{\eta} * \boldsymbol{\lambda}$ we can write

$$
\begin{aligned}
& Z_{d}(x)=d^{-(u+v+z)} \sum_{d=d_{1} d_{2}} \\
& \sum_{\substack{0<|r| \leq R / d \\
0<|g| \leq G / d}} \sum_{\substack{\left(a_{1}, a_{2}, b \\
\left(a_{1} a_{2}, b\right)=1 \\
\left(a_{1}, d_{2}\right)=1\right.}}\left(a_{1} a_{2}\right)^{-u} b^{-(v+w)} r^{-z} \boldsymbol{\eta}_{d_{1} a_{1}} \boldsymbol{\lambda}_{d_{2} a_{2}} \boldsymbol{\beta}_{d b} e\left(\frac{-q r g \overline{a_{1} a_{2}}}{b}+g x\right) W_{1}\left(\frac{b x}{M}\right) .
\end{aligned}
$$

Applying Lemma 6 of [11], which is essentially the same as the result of [7], to the above expression with

$$
U \leftrightarrow \frac{B}{d}, \quad K \leftrightarrow \frac{R G}{d^{2}} \asymp \frac{q^{\varepsilon}(A M+B N)^{2}}{d^{2} q M N}, \quad S \leftrightarrow \frac{A_{1}}{d_{1}} \quad \text { and } \quad T \leftrightarrow \frac{A_{2}}{d_{2}}
$$

leads to

$$
\begin{aligned}
Z_{d}(x) & \ll_{\varepsilon} q^{\varepsilon} \sum_{d=d_{1} d_{2}} U(K S T)^{3 / 4}\left(K^{1 / 4}+S^{1 / 4}+T^{1 / 4}\right) \\
& \ll_{\varepsilon} d^{-13 / 4} q^{-3 / 4+\varepsilon} \sum_{d=d_{1} d_{2}} \frac{A^{3 / 4} B(A M+B N)^{3 / 2}}{(M N)^{3 / 4}}\left(\frac{(A M+B N)^{1 / 2}}{d^{1 / 2}(q M N)^{1 / 4}}+\frac{A_{1}^{1 / 4}}{d_{1}^{1 / 4}}+\frac{A_{2}^{1 / 4}}{d_{2}^{1 / 4}}\right) .
\end{aligned}
$$


Plugging this into (3.17) we obtain the proposition.

The following proposition is required for Theorem 1.3.

Proposition 3.4. Assume the conditions of Proposition 3.3. Also assume that $\boldsymbol{\alpha}=$ $\boldsymbol{\eta} * \boldsymbol{\lambda}, \boldsymbol{\beta}=\boldsymbol{\nu} * \boldsymbol{\xi}$, where the sequences $\boldsymbol{\eta}_{a_{1}}, \boldsymbol{\lambda}_{a_{2}}, \boldsymbol{\nu}_{b_{1}}, \boldsymbol{\xi}_{b_{2}}$ are supported on the intervals $\left[A_{1}, 2 A_{1}\right],\left[A_{2}, 2 A_{2}\right],\left[B_{1}, 2 B_{1}\right]$ and $\left[B_{2}, 2 B_{2}\right]$ with $A=A_{1} A_{2}, B=B_{1} B_{2}$, and satisfy $\boldsymbol{\eta}_{a}, \boldsymbol{\lambda}_{a} \ll a^{\varepsilon}, \boldsymbol{\nu}_{b}, \boldsymbol{\xi}_{b} \ll b^{\varepsilon}$. Suppose further that

$$
\boldsymbol{\eta}_{a_{1}}=\eta\left(\frac{a_{1}}{A_{1}}\right) \quad \text { and } \quad \boldsymbol{\nu}_{b_{1}}=\nu\left(\frac{b_{1}}{B_{1}}\right)
$$

where $\eta$ and $\nu$ are smooth functions supported in $[1,2]$ such that $\eta^{(j)}, \nu^{(j)} \ll_{j} q^{\varepsilon}$ for any fixed $j \geq 0$. Then

$$
\mathcal{S}=\mathcal{M}_{1}^{+}+\mathcal{M}_{1}^{-}+\mathcal{E},
$$

where $\mathcal{M}_{1}^{ \pm}$are defined as in (3.3) and

$$
\begin{aligned}
\mathcal{E} \ll_{\varepsilon} q^{\vartheta-1 / 2+\varepsilon}(A B)^{-3 / 4}(A M+B N)^{3 / 2}(M N)^{-3 / 4} & \\
& \left(A_{2} B_{2}+\frac{(A M+B N)^{2}}{q M N}\right)^{1 / 2}\left(A_{2} B_{2}+\frac{B}{A_{1}}\right)^{1 / 2}
\end{aligned}
$$

with $\vartheta=7 / 64$.

Proof. We proceed from (3.8),

$$
\mathcal{S}=\mathcal{M}_{1}^{+}+\mathcal{M}_{1}^{-}+\mathcal{E}+O_{C}\left(q^{-C}\right)
$$

with

$$
\begin{aligned}
& \mathcal{E} \ll \frac{1}{\sqrt{A B M N}} \sum_{d \leq R} \int_{(\varepsilon)} \int_{(\varepsilon)} \int_{(\varepsilon)} \int_{(\varepsilon)} \int_{x \asymp d M N /(A M+B N)} \\
& x^{-\operatorname{Re}(w)}\left|\widehat{f_{ \pm}}(u, v, w, z)\right|\left|Z_{d}(x)\right| d x d u d v d w d z,
\end{aligned}
$$

where

$$
Z_{d}(x)=d^{w-z} \sum_{\substack{0<|r| \leq R / d \\ 0<|g| \leq G / d}} \sum_{(a, b)=d} a^{-u} b^{-(v+w)} r^{-z} \boldsymbol{\alpha}_{a} \boldsymbol{\beta}_{b} e\left(\frac{-q r g \bar{a} / d}{b / d}+g x\right) W_{1}\left(\frac{b x}{d M}\right) .
$$

Since $\boldsymbol{\alpha}=\boldsymbol{\eta} * \boldsymbol{\lambda}$ and $\boldsymbol{\beta}=\boldsymbol{\nu} * \boldsymbol{\xi}$, we get

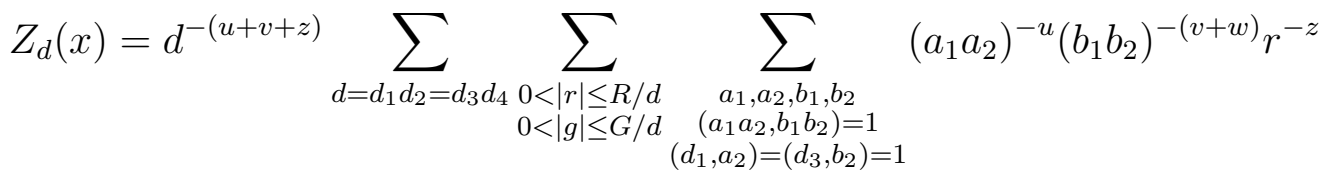

$$
\begin{aligned}
& \eta\left(\frac{d_{1} a_{1}}{A_{1}}\right) \nu\left(\frac{d_{3} b_{1}}{B_{1}}\right) \boldsymbol{\lambda}_{d_{2} a_{2}} \boldsymbol{\xi}_{d_{4} b_{2}} e\left(\frac{-q r g \overline{a_{1} a_{2}}}{b_{1} b_{2}}+g x\right) W_{1}\left(\frac{b_{1} b_{2} x}{M}\right) .
\end{aligned}
$$

Note that if we apply Weil's bound on the sum over $a_{1}$ here, we would obtain a slightly weaker result. Instead we apply the Poisson summation formula over $a_{1}$ and obtain

$$
\sum_{\left(a_{1}, b_{1} b_{2}\right)=1} a_{1}^{-u} \eta\left(\frac{d_{1} a_{1}}{A_{1}}\right) e\left(\frac{-q r g \overline{a_{1} a_{2}}}{b_{1} b_{2}}\right)=\sum_{x\left(\bmod b_{1} b_{2}\right)}^{*} e\left(\frac{-q r g \overline{x a_{2}}}{b_{1} b_{2}}\right) \sum_{a_{1} \equiv x\left(\bmod b_{1} b_{2}\right)} a_{1}^{-u} \eta\left(\frac{d_{1} a_{1}}{A_{1}}\right)
$$




$$
=\sum_{k \in \mathbb{Z}} S\left(q r g \overline{a_{2}}, k ; b_{1} b_{2}\right) \int\left(b_{1} b_{2} y\right)^{-u} \eta\left(\frac{d_{1} b_{1} b_{2} y}{A_{1}}\right) e(k y) d y .
$$

The integral is over $y \asymp d_{2} A_{1} / B$, and as before we may restrict the sum to $|k| \leq K$, where

$$
K=\frac{q^{\varepsilon} B}{d_{2} A_{1}} .
$$

Since $\left|S\left(q r g \overline{a_{2}}, 0 ; b_{1} b_{2}\right)\right| \leq\left(q r g, b_{1} b_{2}\right)$, the contribution of the term $k=0$ to $Z_{d}(x)$ is

$$
\begin{aligned}
& \ll_{\varepsilon} \frac{q^{\varepsilon} A_{1}}{B} \sum_{d=d_{1} d_{2}=d_{3} d_{4}} \sum_{\begin{array}{r}
0<|r| \leq R / d \\
0<|g| \leq G / d
\end{array}} \sum_{\substack{a_{2}, b_{1}, b_{2} \\
\left(a_{2}, b_{1} b_{2}\right)=1 \\
\left(d_{1}, a_{2}\right)=\left(d_{3}, b_{2}\right)=1}}\left|\nu\left(\frac{d_{3} b_{1}}{B_{1}}\right) \boldsymbol{\lambda}_{d_{2} a_{2}} \boldsymbol{\xi}_{d_{4} b_{2}}\right|\left(r g, b_{1} b_{2}\right) d_{2} \\
& \ll_{\varepsilon} d^{-3} q^{\varepsilon} A R G \ll_{\varepsilon} d^{-3} q^{-1+\varepsilon} \frac{A(A M+B N)^{2}}{M N} .
\end{aligned}
$$

Hence

$$
Z_{d}(x) \ll_{\varepsilon} q^{\varepsilon} \sum_{d=d_{1} d_{2}=d_{3} d_{4}} \int_{y \asymp d_{2} A_{1} / B} y^{-\operatorname{Re}(u)}|R(y)| d y+d^{-3} q^{-1+\varepsilon} \frac{A(A M+B N)^{2}}{M N},
$$

where

$$
\begin{aligned}
R(y)= & \sum_{\substack{0<|r| \leq R / d \\
0<|g| \leq G / d}} \sum_{\begin{array}{c}
a_{2}, b_{1}, b_{2} \\
\left(a_{2}, b_{1} b_{2}\right)=1 \\
\left(d_{1}, a_{2}\right)=\left(d_{3}, b_{2}\right)=1
\end{array}} \sum_{0<|k| \leq K} S\left(q r g \overline{a_{2}}, k ; b_{1} b_{2}\right) \\
& a_{2}^{-u}\left(b_{1} b_{2}\right)^{-(u+v+w)} r^{-z} \nu\left(\frac{d_{3} b_{1}}{B_{1}}\right) \boldsymbol{\lambda}_{d_{2} a_{2}} \boldsymbol{\xi}_{d_{4} b_{2}} W_{1}\left(\frac{b_{1} b_{2} x}{M}\right) \eta\left(\frac{d_{1} b_{1} b_{2} y}{A_{1}}\right) e(g x+k y) .
\end{aligned}
$$

We now use the following result, which is a special case of Lemma 4.1 of Watt [21].

Lemma 3.5. Let $1 \leq N, R, S, U, W<q, Z \in \mathbb{R}$ and let $\boldsymbol{\alpha}_{r}, \boldsymbol{\beta}_{s}, \boldsymbol{\gamma}_{u}$ be three sequences of complex numbers supported on $[R, 2 R],[S, 2 S]$ and $[1, U]$, and satisfy $\boldsymbol{\alpha}_{r} \ll R^{\varepsilon}, \boldsymbol{\beta}_{s} \ll$ $S^{\varepsilon}, \gamma_{u} \ll U^{\varepsilon}$. For any $r \in[R, 2 R]$ and $s \in[S, 2 S]$, suppose that $F_{r, s}$ is a five times continuously differentiable function supported in $[1,2]$ such that $F_{r, s}^{(j)} \ll_{j} q^{\varepsilon}$ for any $0 \leq j \leq 5$. Then

$$
\begin{aligned}
\sum_{(r, s)=1} \sum_{\substack{0<u \leq U \\
0<w \leq W}} \sum_{n} \boldsymbol{\alpha}_{r} \boldsymbol{\beta}_{s} \boldsymbol{\gamma}_{u} e(Z w) F_{r, s}\left(\frac{n}{N}\right) S(q u \bar{r}, w ; n s) \\
\quad \ll_{\varepsilon} q^{\vartheta}(1+Z W) N S \sqrt{R U W}(R S+U)^{1 / 2}(R S+W)^{1 / 2}\left(X^{2 \vartheta}+X^{-3 / 2}\right),
\end{aligned}
$$

where $\vartheta=7 / 64$ and

$$
X=\frac{N S \sqrt{R}}{\sqrt{q U W}} .
$$

Separating the variables $b_{1}, b_{2}$ in $W_{1}$ and $\eta$ in (3.20) using their Mellin transforms, and then applying the above lemma with

$$
\begin{gathered}
R \leftrightarrow \frac{A_{2}}{d_{2}}, \quad S \leftrightarrow \frac{B_{2}}{d_{4}}, \quad U \leftrightarrow \frac{R G}{d^{2}} \asymp \frac{q^{\varepsilon}(A M+B N)^{2}}{d^{2} q M N}, \quad W \leftrightarrow K \asymp \frac{q^{\varepsilon} B}{d_{2} A_{1}}, \\
N \leftrightarrow \frac{B_{1}}{d_{3}}, \quad Z \leftrightarrow \frac{d_{2} A_{1}}{B} \quad \text { and } \quad X \leftrightarrow \frac{\sqrt{A B M N}}{A M+B N}(\ll 1),
\end{gathered}
$$


we get

$$
\begin{aligned}
R(y) \ll_{\varepsilon} q^{\vartheta+\varepsilon} \frac{B}{d} \sqrt{\frac{(A M+B N)^{2} A_{2} B}{d^{2} d_{2}^{2} q A_{1} M N}}\left(\frac{A_{2} B_{2}}{d_{2} d_{4}}+\frac{(A M+B N)^{2}}{d^{2} q M N}\right)^{1 / 2} \\
\left(\frac{A_{2} B_{2}}{d_{2} d_{4}}+\frac{B}{d_{2} A_{1}}\right)^{1 / 2} \frac{(A M+B N)^{3 / 2}}{(A B M N)^{3 / 4}} \\
\ll_{\varepsilon} d^{-2} q^{\vartheta-1 / 2+\varepsilon} \frac{B}{d_{2} A_{1}} \frac{(A M+B N)^{5 / 2}}{(A B)^{1 / 4}(M N)^{5 / 4}} \\
\left(\frac{A_{2} B_{2}}{d_{2} d_{4}}+\frac{(A M+B N)^{2}}{d^{2} q M N}\right)^{1 / 2}\left(\frac{A_{2} B_{2}}{d_{2} d_{4}}+\frac{B}{d_{2} A_{1}}\right)^{1 / 2} .
\end{aligned}
$$

Plugging this into (3.19) yields

$$
\begin{gathered}
Z_{d}(x) \ll_{\varepsilon} d^{-2} q^{\vartheta-1 / 2+\varepsilon} \frac{(A M+B N)^{5 / 2}}{(A B)^{1 / 4}(M N)^{5 / 4}}\left(A_{2} B_{2}+\frac{(A M+B N)^{2}}{d^{2} q M N}\right)^{1 / 2}\left(A_{2} B_{2}+\frac{B}{A_{1}}\right)^{1 / 2} \\
+d^{-3} q^{-1+\varepsilon} \frac{A(A M+B N)^{2}}{M N}
\end{gathered}
$$

and hence

$$
\mathcal{E} \ll_{\varepsilon} q^{\vartheta-1 / 2+\varepsilon} \frac{(A M+B N)^{3 / 2}}{(A B)^{3 / 4}(M N)^{3 / 4}}\left(A_{2} B_{2}+\frac{(A M+B N)^{2}}{q M N}\right)^{1 / 2}\left(A_{2} B_{2}+\frac{B}{A_{1}}\right)^{1 / 2} .
$$

This completes the proof of the proposition.

\section{Proof of Theorem 1.1}

We proceed from $(2.2)$ and start the evaluation of the off-diagonal terms, $S_{\alpha, \beta}^{+}$. We assume that the sequences $\boldsymbol{\alpha}_{a}, \boldsymbol{\beta}_{b}$ are supported on $[A, 2 A]$ and $[B, 2 B]$. We remark that our main term analysis is inspired by the nice papers of Young [22] and Zacharias $[23]$.

4.1. Partition into dyadic intervals. We first apply a dyadic partition of unity to the sums over $m$ and $n$. Let $W$ be a smooth non-negative function supported in $[1,2]$ such that

$$
\sum_{M} W\left(\frac{x}{M}\right)=1
$$

where $M$ runs over a sequence of real numbers with $\#\left\{M: X^{-1} \leq M \leq X\right\} \ll \log X$. With this partition of unity, we write

$$
S_{\alpha, \beta}^{+}=\sum_{M, N} S_{\alpha, \beta}^{+}(M, N),
$$

where

$$
S_{\alpha, \beta}^{+}(M, N)=\sum_{\substack{a m \equiv \pm b n(\bmod q) \\ a m \neq b n}} \frac{\boldsymbol{\alpha}_{a} \boldsymbol{\beta}_{b}}{\sqrt{a b} m^{1 / 2+\alpha} n^{1 / 2+\beta}} W\left(\frac{m}{M}\right) W\left(\frac{n}{N}\right) V_{+}\left(\frac{\pi m n}{q}\right) .
$$

Due to the rapid decay of $V_{+}$in Remark 2.3, we may assume that $M N \ll q^{1+\varepsilon}$. 
Separating the variables $m$ and $n$ in $V_{+}$using (2.1), we have

$$
S_{\alpha, \beta}^{+}(M, N)=\frac{1}{2 \pi i} \int_{(\varepsilon)} X_{+}(s)\left(\frac{q}{\pi}\right)^{s} \sum_{\substack{a \\ a m \pm b n(\bmod q) \\ a m \neq b n}} \frac{\boldsymbol{\alpha}_{a} \boldsymbol{\beta}_{b}}{\sqrt{a b} m^{1 / 2+\alpha+s} n^{1 / 2+\beta+s}} W\left(\frac{m}{M}\right) W\left(\frac{n}{N}\right) \frac{d s}{s} .
$$

We now apply Propositions 3.1 and 3.2 to the above sum: given some small $\delta_{0}>0$, let

$$
\begin{aligned}
& \mathcal{A}_{1}=\left\{(M, N): A B M N \ll q^{2-2 \delta_{0}}\right\}, \\
& \mathcal{A}_{2,<}=\left\{(M, N): A B M N \gg q^{2-2 \delta_{0}}, B M \ll q^{1-6 \delta_{0}+\varepsilon}, A \ll q^{-2 \delta_{0}} N, B \gg q^{2 \delta_{0}} M\right\}, \\
& \mathcal{A}_{2,>}=\left\{(M, N): A B M N \gg q^{2-2 \delta_{0}}, A N \ll q^{1-6 \delta_{0}+\varepsilon}, A \gg q^{2 \delta_{0}} N, B \ll q^{-2 \delta_{0}} M\right\}, \\
& \mathcal{A}_{3}=\left\{(M, N): M N \ll q^{1+\varepsilon}\right\} \backslash\left(\mathcal{A}_{1} \cup \mathcal{A}_{2,<} \cup \mathcal{A}_{2,>}\right) .
\end{aligned}
$$

If $(M, N) \in \mathcal{A}_{1}$ we apply (3.1) of Proposition 3.1; if $(M, N) \in \mathcal{A}_{2,<} \cup \mathcal{A}_{2,>}$ we apply Proposition 3.2; and in the remaining case we apply (3.2) of Proposition 3.1. Then we obtain

$$
\begin{aligned}
S_{\alpha, \beta}^{+}(M, N)=\mathbb{1}_{(M, N) \in \mathcal{A}_{3}}\left(\mathcal{M}_{1}^{+}(M, N)+\mathcal{M}_{1}^{-}(M, N)\right) \\
+\mathbb{1}_{(M, N) \in \mathcal{A}_{2,<}} \mathcal{M}_{2,<}(M, N)+\mathbb{1}_{(M, N) \in \mathcal{A}_{2,>}} \mathcal{M}_{2,>}(M, N)+\mathcal{E}(M, N)
\end{aligned}
$$

with obvious meaning. More work on the secondary main terms $\mathcal{M}_{1}^{ \pm}(M, N)$ and $\mathcal{M}_{2, \gtrless}(M, N)$ is required before we can make any use of them. We shall do that in subsections 4.3 and 4.4 after showing in the next subsection that the error term $\mathcal{E}(M, N)$ is acceptable.

4.2. The error term $\mathcal{E}(M, N)$. Without loss of generality, we may assume that $M \ll$ $N$. From (3.1) of Proposition 3.1 and Proposition 3.2, the error term is $O_{\varepsilon}\left(q^{-\delta_{0}+\varepsilon}\right)$ if $A B M N \ll q^{2-2 \delta_{0}}$, or if

$$
B M \ll q^{1-6 \delta_{0}+\varepsilon}, \quad A \ll q^{-2 \delta_{0}} N \quad \text { and } \quad B \gg q^{2 \delta_{0}} M .
$$

We now consider the remaining case $(M, N) \in \mathcal{A}_{3}$. Note that if $N \gg q^{\kappa+6 \delta_{0}}$, then $M \ll_{\varepsilon} q^{1-\kappa-6 \delta_{0}+\varepsilon}$, and all the conditions in (4.2) are satisfied. To obtain the last bound in (4.2), we make use of the assumption that $A B M N \gg q^{2-2 \delta_{0}}$. This assumption, in combination with the fact that $A$ and $M N$ are bounded by $q^{\kappa}$ and $q^{1+\varepsilon}$ respectively, force $B \gg q^{1-\kappa-2 \delta_{0}-\varepsilon} \gg q^{2 \delta_{0}} M$. So we may assume that $N \ll q^{\kappa+6 \delta_{0}}$. In that case, provided that $\kappa<6 / 11$ (which we subsequently assume), the first term in (3.4) in Proposition 3.1 dominates the second term, i.e.

$$
\begin{aligned}
\mathcal{E}(M, N) & \ll_{\varepsilon} q^{-17 / 20+\varepsilon}(A B)^{-3 / 20}(A M+B N)^{17 / 10}(A+B)^{1 / 4}(M N)^{-17 / 20} \\
& \ll_{\varepsilon} q^{-17 / 20+\varepsilon}(A / B)^{3 / 20} A^{33 / 20}+q^{-17 / 20+\varepsilon} A^{31 / 20} B^{1 / 10} \\
& +q^{-17 / 20+\varepsilon} A^{1 / 10} B^{31 / 20}(N / M)^{17 / 20}+q^{-17 / 20+\varepsilon}(B / A)^{3 / 20} B^{33 / 20}(N / M)^{17 / 20} .
\end{aligned}
$$

Note that as $A B M N \gg q^{2-2 \delta_{0}}$, it follows that $A B \gg q^{1-2 \delta_{0}-\varepsilon}$. With both $A$ and $B$ bounded by $q^{\kappa}$, one sees that $q^{1-2 \kappa-2 \delta_{0}-\varepsilon} \ll A / B \ll q^{2 \kappa+2 \delta_{0}-1+\varepsilon}$.

We divide into two cases:

Case 1: $B M \gg q^{1-6 \delta_{0}+\varepsilon}$ or $B \ll q^{2 \delta_{0}} M$. Then, using as above the assumption $A B M N \gg q^{2-2 \delta_{0}}$ in combination with the fact that $A, B$ and $M N$ are bounded by $q^{\kappa}$, 
$q^{\kappa}$ and $q^{1+\varepsilon}$ respectively, it follows that $M \gg q^{1-\kappa-6 \delta_{0}}$. So $1 \leq N / M \ll q^{2 \kappa+12 \delta_{0}-1+\varepsilon}$. Therefore

$$
\mathcal{E}(M, N) \ll_{\varepsilon} q^{-17 / 20+3\left(2 \kappa+2 \delta_{0}-1\right) / 20+33 \kappa / 20+17\left(2 \kappa+12 \delta_{0}-1\right) / 20+\varepsilon}=q^{-37 / 20+73 \kappa / 20+21 \delta_{0} / 2+\varepsilon} ;
$$

Case 2: $A \gg q^{-2 \delta_{0}} N$. As $M \gg q^{2-2 \delta_{0}} / A B N$ we have

$$
\begin{aligned}
\mathcal{E}(M, N) & \ll_{\varepsilon} q^{-17 / 20+3\left(2 \kappa+2 \delta_{0}-1\right) / 20+33 \kappa / 20+\varepsilon}+q^{-51 / 20+17 \delta_{0} / 10+\varepsilon} A^{19 / 20} B^{12 / 5} N^{17 / 10} \\
& +q^{-51 / 20+17 \delta_{0} / 10+\varepsilon} A^{7 / 10} B^{53 / 20} N^{17 / 10} \\
& \ll_{\varepsilon} q^{-1+39 \kappa / 20+3 \delta_{0} / 10+\varepsilon}+q^{-51 / 20+101 \kappa / 20+51 \delta_{0} / 10+\varepsilon} .
\end{aligned}
$$

Summing up we obtain

$\mathcal{E}(M, N) \ll_{\varepsilon} q^{-\delta_{0}+\varepsilon}+q^{-37 / 20+73 \kappa / 20+21 \delta_{0} / 2+\varepsilon}+q^{-1+39 \kappa / 20+3 \delta_{0} / 10+\varepsilon}+q^{-51 / 20+101 \kappa / 20+51 \delta_{0} / 10+\varepsilon}$, provided that $\kappa<6 / 11$. We hence conclude that $\mathcal{E}(M, N) \ll_{\varepsilon} q^{-\delta_{0}+\varepsilon}$ for some $\delta_{0}>0$ if $\kappa<51 / 101$.

4.3. The secondary main terms $\mathcal{M}_{1}^{ \pm}(M, N)$. For $(M, N) \in \mathcal{A}_{3}$, from (3.2) of Proposition 3.1 we have

$$
\begin{aligned}
\mathcal{M}_{1}^{+}(M, N)= & \sum_{r \neq 0} \sum_{\substack{d \\
(a, b)=1}} \frac{\boldsymbol{\alpha}_{d a} \boldsymbol{\beta}_{d b}}{d \sqrt{a b}} \frac{1}{2 \pi i} \int_{(\varepsilon)} X_{+}(s)\left(\frac{q}{\pi}\right)^{s} \\
& \int(b x)^{-(1 / 2+\alpha+s)}(a x-q r / b)^{-(1 / 2+\beta+s)} W\left(\frac{b x}{M}\right) W\left(\frac{a b x-q r}{b N}\right) d x \frac{d s}{s}
\end{aligned}
$$

and

$$
\begin{aligned}
\mathcal{M}_{1}^{-}(M, N)= & \sum_{r \neq 0} \sum_{\substack{d \\
(a, b)=1}} \frac{\boldsymbol{\alpha}_{d a} \boldsymbol{\beta}_{d b}}{d \sqrt{a b}} \frac{1}{2 \pi i} \int_{(\varepsilon)} X_{+}(s)\left(\frac{q}{\pi}\right)^{s} \\
& \int(b x)^{-(1 / 2+\alpha+s)}(q r / b-a x)^{-(1 / 2+\beta+s)} W\left(\frac{b x}{M}\right) W\left(\frac{q r-a b x}{b N}\right) d x \frac{d s}{s} .
\end{aligned}
$$

Writing $W$ in terms of its Mellin transform we get

$$
\begin{aligned}
& \mathcal{M}_{1}^{+}(M, N)=\frac{1}{(2 \pi i)^{3}} \int_{(\varepsilon)} \int_{\left(c_{2}\right)} \int_{\left(c_{1}\right)} X_{+}(s) \widetilde{W}(u) \widetilde{W}(v)\left(\frac{q}{\pi}\right)^{s} M^{u} N^{v} \\
& \sum_{r \neq 0} \sum_{\substack{d \\
(a, b)=1}} \frac{\boldsymbol{\alpha}_{d a} \boldsymbol{\beta}_{d b}}{d a^{1+\beta+s+v} b^{1+\alpha+s+u}} \int x^{-(1 / 2+\alpha+s+u)}(x-q r / a b)^{-(1 / 2+\beta+s+v)} d x d u d v \frac{d s}{s} .
\end{aligned}
$$

Note that if $r>0$ then the integral over $x$ is restricted to $x>q r / a b$, and if $r<0$ then it is restricted to $x>0$. For absolute convergence, we also need to impose the conditions

$$
\begin{cases}\operatorname{Re}(\alpha+\beta+2 s+u+v)>0, \operatorname{Re}(\beta+s+v)<1 / 2 & \text { if } r>0, \\ \operatorname{Re}(\alpha+\beta+2 s+u+v)>0, \operatorname{Re}(\alpha+s+u)<1 / 2 & \text { if } r<0 .\end{cases}
$$

Under these assumptions, the $x$-integral is equal to (see, for instance, 17.43.21 and 17.43 .22 of $[8])$

$$
\left(\frac{q|r|}{a b}\right)^{-(\alpha+\beta+2 s+u+v)} \times \begin{cases}\frac{\Gamma(\alpha+\beta+2 s+u+v) \Gamma(1 / 2-\beta-s-v)}{\Gamma(1 / 2+\alpha+s+u)} & \text { if } r>0, \\ \frac{\Gamma(\alpha+\beta+2 s+u+v) \Gamma(1 / 2-\alpha-s-u)}{\Gamma(1 / 2+\beta+s+v)} & \text { if } r<0 .\end{cases}
$$


Hence

$$
\begin{aligned}
\mathcal{M}_{1}^{+}(M, N)= & \sum_{r \geq 1} \sum_{\substack{d \\
(a, b)=1}} \frac{\boldsymbol{\alpha}_{d a} \boldsymbol{\beta}_{d b}}{d a b} \frac{1}{(2 \pi i)^{3}} \int_{\left(c_{2}\right)} \int_{\left(c_{1}\right)} \widetilde{W}(u) \widetilde{W}(v) M^{u} N^{v} \\
& \int_{(\varepsilon)} X_{+}(s) H^{+}(s)\left(\frac{q}{\pi}\right)^{s}\left(\frac{q}{a}\right)^{-(\alpha+s+u)}\left(\frac{q}{b}\right)^{-(\beta+s+v)} r^{-(\alpha+\beta+2 s+u+v)} \frac{d s}{s} d u d v,
\end{aligned}
$$

where

$$
H^{+}(s)=\Gamma(\alpha+\beta+2 s+u+v)\left(\frac{\Gamma(1 / 2-\beta-s-v)}{\Gamma(1 / 2+\alpha+s+u)}+\frac{\Gamma(1 / 2-\alpha-s-u)}{\Gamma(1 / 2+\beta+s+v)}\right) .
$$

A similar formula holds for $\mathcal{M}_{1}^{-}(M, N)$ with $H^{+}(s)$ being replaced by $H^{-}(s)$, where

$$
H^{-}(s)=\frac{\Gamma(1 / 2-\alpha-s-u) \Gamma(1 / 2-\beta-s-v)}{\Gamma(1-\alpha-\beta-2 s-u-v)},
$$

and the imposed conditions for the absolute convergence are

$$
\operatorname{Re}(\alpha+s+u)<1 / 2, \quad \operatorname{Re}(\beta+s+v)<1 / 2 .
$$

To keep the symmetry, we write

$$
\mathcal{M}_{1}^{+}(M, N)+\mathcal{M}_{1}^{-}(M, N)=\mathcal{P}_{1}(M, N)+\mathcal{P}_{2}(M, N),
$$

where, for $j=1,2$,

$$
\begin{aligned}
\mathcal{P}_{j}(M, N)= & \sum_{r \geq 1} \sum_{\substack{d \\
(a, b)=1}} \frac{\boldsymbol{\alpha}_{d a} \boldsymbol{\beta}_{d b}}{d a b} \frac{1}{(2 \pi i)^{3}} \int_{\left(c_{2}\right)} \int_{\left(c_{1}\right)} \widetilde{W}(u) \widetilde{W}(v) M^{u} N^{v} \\
& \int_{(\varepsilon)} X_{+}(s) H_{j}(s)\left(\frac{q}{\pi}\right)^{s}\left(\frac{q}{a}\right)^{-(\alpha+s+u)}\left(\frac{q}{b}\right)^{-(\beta+s+v)} r^{-(\alpha+\beta+2 s+u+v)} \frac{d s}{s} d u d v
\end{aligned}
$$

where

$$
H_{1}(s)=\Gamma(1 / 2-\beta-s-v)\left(\frac{\Gamma(\alpha+\beta+2 s+u+v)}{\Gamma(1 / 2+\alpha+s+u)}+\frac{\Gamma(1 / 2-\alpha-s-u)}{2 \Gamma(1-\alpha-\beta-2 s-u-v)}\right)
$$

and

$$
H_{2}(s)=\Gamma(1 / 2-\alpha-s-u)\left(\frac{\Gamma(\alpha+\beta+2 s+u+v)}{\Gamma(1 / 2+\beta+s+v)}+\frac{\Gamma(1 / 2-\beta-s-v)}{2 \Gamma(1-\alpha-\beta-2 s-u-v)}\right) .
$$

4.3.1. The $r$-sum. The aim here is to show that we can somehow replace the sum over $r$ in (4.5) by $\zeta(\alpha+\beta+2 s+u+v)$.

We start with $\mathcal{P}_{1}(M, N)$. Choose $c_{1}=0$ and $c_{2}=\varepsilon$. We move the $s$-contour to the right to $\operatorname{Re}(s)=1 / 2-\varepsilon / 3$, crossing a simple pole at $s=1 / 2-\beta-v$ from the first gamma factor of $H_{1}(s)$. In doing so we obtain

$$
\mathcal{P}_{1}(M, N)=\mathcal{P}_{1}^{\prime}(M, N)-\mathcal{R}_{1}(M, N),
$$

where $\mathcal{P}_{1}^{\prime}(M, N)$ is the integral along the new line and

$$
\begin{array}{r}
\mathcal{R}_{1}(M, N)=-\frac{3}{2} \sum_{r \geq 1} \sum_{\substack{d \\
(a, b)=1}} \frac{\boldsymbol{\alpha}_{d a} \boldsymbol{\beta}_{d b}}{d a b} \frac{1}{(2 \pi i)^{2}} \int_{(\varepsilon)} \int_{(0)} X_{+}(1 / 2-\beta-v) \widetilde{W}(u) \widetilde{W}(v) M^{u} N^{v} \\
\left(\frac{q}{\pi}\right)^{1 / 2-\beta-v}\left(\frac{q}{a}\right)^{-(1 / 2+\alpha-\beta+u-v)}\left(\frac{q}{b}\right)^{-1 / 2} r^{-(1+\alpha-\beta+u-v)} \frac{d u d v}{1 / 2-\beta-v} .
\end{array}
$$


As moving the $u$-contour in the above expression to $\operatorname{Re}(u)=2 \varepsilon$ encountering no pole, we have

$$
\begin{array}{r}
\mathcal{R}_{1}(M, N)=-\frac{3}{2} \sum_{\substack{d \\
(a, b)=1}} \frac{\boldsymbol{\alpha}_{d a} \boldsymbol{\beta}_{d b}}{d a b} \frac{1}{(2 \pi i)^{2}} \int_{(\varepsilon)} \int_{(2 \varepsilon)} X_{+}(1 / 2-\beta-v) \widetilde{W}(u) \widetilde{W}(v) M^{u} N^{v} \\
\left(\frac{q}{\pi}\right)^{1 / 2-\beta-v}\left(\frac{q}{a}\right)^{-(1 / 2+\alpha-\beta+u-v)}\left(\frac{q}{b}\right)^{-1 / 2} \zeta(1+\alpha-\beta+u-v) \frac{d u d v}{1 / 2-\beta-v}
\end{array}
$$

With $\mathcal{P}_{1}^{\prime}(M, N)$, the $r$-sum can be written as $\zeta(\alpha+\beta+2 s+u+v)$. We then shift the $s$-contour back to $\operatorname{Re}(s)=\varepsilon$, but this time crossing two simple poles at $s=1 / 2-\beta-v$, again, and $s=1 / 2-(\alpha+\beta+u+v) / 2$, because of the zeta-function. We write

$$
\mathcal{P}_{1}^{\prime}(M, N)=\mathcal{P}_{1}^{\prime \prime}(M, N)+\mathcal{R}_{1}^{\prime}(M, N)+\mathcal{R}_{1}^{\prime \prime}(M, N)
$$

accordingly, where $\mathcal{R}_{1}^{\prime}(M, N)$ and $\mathcal{R}_{1}^{\prime \prime}(M, N)$ are the residues at $s=1 / 2-\beta-v$ and $s=1 / 2-(\alpha+\beta+u+v) / 2$, respectively. It follows that

$$
\mathcal{P}_{1}(M, N)=\mathcal{P}_{1}^{\prime \prime}(M, N)-\left(\mathcal{R}_{1}(M, N)-\mathcal{R}_{1}^{\prime}(M, N)\right)+\mathcal{R}_{1}^{\prime \prime}(M, N) .
$$

Note that $\mathcal{R}_{1}^{\prime}(M, N)$ is the same as $\mathcal{R}_{1}(M, N)$ in (4.6) but with the $u$-contour being along $\operatorname{Re}(u)=0$. So the difference $\mathcal{R}_{1}(M, N)-\mathcal{R}_{1}^{\prime}(M, N)$ is the residue at $u=v-\alpha+\beta$ of (4.6). That is to say

$$
\begin{aligned}
\mathcal{R}_{1}(M, N)-\mathcal{R}_{1}^{\prime}(M, N) & =-\frac{3}{2 q} \sum_{a, b} \frac{\boldsymbol{\alpha}_{a} \boldsymbol{\beta}_{b}}{\sqrt{a b}} \\
& \frac{1}{2 \pi i} \int_{(\varepsilon)} X_{+}(1 / 2-\beta-v) \widetilde{W}(v-\alpha+\beta) \widetilde{W}(v)\left(\frac{q}{\pi}\right)^{1 / 2-\beta-v} M^{v-\alpha+\beta} N^{v} \frac{d v}{1 / 2-\beta-v} .
\end{aligned}
$$

We shall make use of the fact that

$$
\begin{aligned}
\widetilde{W}(v) N^{v} & =N^{v} \int_{0}^{\infty} x^{v-1} W(x) d x \\
& =\sum_{n} n^{v-1} W\left(\frac{n}{N}\right)+N^{v} \sum_{n} \int_{n / N}^{(n+1) / N}\left(x^{v-1} W(x)-\left(\frac{n}{N}\right)^{v-1} W\left(\frac{n}{N}\right)\right) d x \\
& =\sum_{n} n^{v-1} W\left(\frac{n}{N}\right)+O_{\varepsilon}\left(q^{\varepsilon} N^{\operatorname{Re}(v)-1}|v|\right) .
\end{aligned}
$$

Using this and (2.1) we obtain

$$
\begin{aligned}
\mathcal{R}_{1}(M, N) & -\mathcal{R}_{1}^{\prime}(M, N) \\
= & -\frac{3}{2 q} \sum_{\substack{a, b \\
m, n}} \frac{\boldsymbol{\alpha}_{a} \boldsymbol{\beta}_{b}}{\sqrt{a b} m^{1 / 2+\alpha} n^{1 / 2+\beta}} W\left(\frac{m}{M}\right) W\left(\frac{n}{N}\right) V_{+}\left(\frac{\pi m n}{q}\right)+O_{\varepsilon}\left(q^{\kappa-1 / 2+\varepsilon}(M N)^{-1}\right) .
\end{aligned}
$$

For $\mathcal{R}_{1}^{\prime \prime}(M, N)$, we have

$$
H_{1}\left(\frac{1-\alpha-\beta-u-v}{2}\right)=\frac{2}{\alpha-\beta+u-v} .
$$

Hence

$$
\mathcal{R}_{1}^{\prime \prime}(M, N)=\frac{2}{q} \sum_{\substack{d \\(a, b)=1}} \frac{\boldsymbol{\alpha}_{d a} \boldsymbol{\beta}_{d b}}{d \sqrt{a b}} \frac{1}{(2 \pi i)^{2}} \int_{(\varepsilon)} \int_{(0)} X_{+}\left(\frac{1-\alpha-\beta-u-v}{2}\right) \widetilde{W}(u) \widetilde{W}(v) M^{u} N^{v}
$$




$$
\left(\frac{q}{\pi}\right)^{(1-\alpha-\beta-u-v) / 2}\left(\frac{a}{b}\right)^{(\alpha-\beta+u-v) / 2} \frac{d u d v}{(1-\alpha-\beta-u-v)(\alpha-\beta+u-v)} .
$$

We apply the same argument to $\mathcal{P}_{2}(M, N)$, but this time choose $c_{1}=\varepsilon$ and $c_{2}=0$. Similarly we obtain

$$
\mathcal{P}_{2}(M, N)=\mathcal{P}_{2}^{\prime \prime}(M, N)-\left(\mathcal{R}_{2}(M, N)-\mathcal{R}_{2}^{\prime}(M, N)\right)+\mathcal{R}_{2}^{\prime \prime}(M, N) .
$$

In particular, note that

$$
H_{2}\left(\frac{1-\alpha-\beta-u-v}{2}\right)=-\frac{2}{\alpha-\beta+u-v} .
$$

So

$$
\begin{gathered}
\mathcal{R}_{2}^{\prime \prime}(M, N)=-\frac{2}{q} \sum_{\substack{d \\
(a, b)=1}} \frac{\boldsymbol{\alpha}_{d a} \boldsymbol{\beta}_{d b}}{d \sqrt{a b}} \frac{1}{(2 \pi i)^{2}} \int_{(0)} \int_{(\varepsilon)} X_{+}\left(\frac{1-\alpha-\beta-u-v}{2}\right) \widetilde{W}(u) \widetilde{W}(v) M^{u} N^{v} \\
\left(\frac{q}{\pi}\right)^{(1-\alpha-\beta-u-v) / 2}\left(\frac{a}{b}\right)^{(\alpha-\beta+u-v) / 2} \frac{d u d v}{(1-\alpha-\beta-u-v)(\alpha-\beta+u-v)} .
\end{gathered}
$$

Apart from the minus sign and the changes of the contours, this is the same as $\mathcal{R}_{1}^{\prime \prime}(M, N)$. Hence, using (4.7) as before,

$$
\begin{aligned}
\mathcal{R}_{1}^{\prime \prime}(M, N)+\mathcal{R}_{2}^{\prime \prime}(M, N)=-\operatorname{Res}_{u=v-\alpha+\beta} \text { of }(4.8) \\
=-\frac{1}{q} \sum_{a, b} \frac{\boldsymbol{\alpha}_{a} \boldsymbol{\beta}_{b}}{\sqrt{a b}} \frac{1}{2 \pi i} \int_{(\varepsilon)} X_{+}(1 / 2-\beta-v)\left(\frac{q}{\pi}\right)^{1 / 2-\beta-v} \\
\widetilde{W}(1 / 2-\beta-v) \widetilde{W}(v) M^{1 / 2-\beta-v} N^{v} \frac{d v}{(1 / 2-\beta-v)} \\
=-\frac{1}{q} \sum_{\substack{a, b \\
m, n}} \frac{\boldsymbol{\alpha}_{a} \boldsymbol{\beta}_{b}}{\sqrt{a b} m^{1 / 2+\alpha} n^{1 / 2+\beta}} W\left(\frac{m}{M}\right) W\left(\frac{n}{N}\right) V_{+}\left(\frac{\pi m n}{q}\right)+O_{\varepsilon}\left(q^{\kappa-1 / 2+\varepsilon} M^{-1 / 2} N^{-1}\right) .
\end{aligned}
$$

We sum up the calculations in this subsection in the following proposition.

Proposition 4.1. For $(M, N) \in \mathcal{A}_{3}$ we have

$$
\begin{aligned}
\mathcal{M}_{1}^{+}(M, N) & +\mathcal{M}_{1}^{-}(M, N)=\mathcal{P}_{1}^{\prime \prime}(M, N)+\mathcal{P}_{2}^{\prime \prime}(M, N) \\
& +\frac{2}{q} \sum_{\substack{a, b \\
m, n}} \frac{\boldsymbol{\alpha}_{a} \boldsymbol{\beta}_{b}}{\sqrt{a b} m^{1 / 2+\alpha} n^{1 / 2+\beta}} W\left(\frac{m}{M}\right) W\left(\frac{n}{N}\right) V_{+}\left(\frac{\pi m n}{q}\right)+O_{\varepsilon}\left(q^{7 \kappa / 2+5 \delta_{0}-5 / 2+\varepsilon}\right),
\end{aligned}
$$

where, for $j=1,2$,

$$
\begin{aligned}
\mathcal{P}_{j}^{\prime \prime}(M, N) & =\sum_{\substack{d \\
(a, b)=1}} \frac{\boldsymbol{\alpha}_{d a} \boldsymbol{\beta}_{d b}}{d a b} \frac{1}{(2 \pi i)^{3}} \int_{\left(c_{2}\right)} \int_{\left(c_{1}\right)} \widetilde{W}(u) \widetilde{W}(v) M^{u} N^{v} \\
& \int_{(\varepsilon)} X_{+}(s) H_{j}(s)\left(\frac{q}{\pi}\right)^{s}\left(\frac{q}{a}\right)^{-(\alpha+s+u)}\left(\frac{q}{b}\right)^{-(\beta+s+v)} \zeta(\alpha+\beta+2 s+u+v) \frac{d s}{s} d u d v .
\end{aligned}
$$


4.4. The secondary main terms $\mathcal{M}_{2, \gtrless}(M, N)$. Applying Proposition 3.2 to $S_{\alpha, \beta}^{+}(M, N)$ for the pairs $(M, N) \in \mathcal{A}_{2,<}$ we get

$$
\begin{aligned}
\mathcal{M}_{2,<}(M, N)=\frac{2}{q} \sum_{a, b} \frac{\boldsymbol{\alpha}_{a} \boldsymbol{\beta}_{b}}{\sqrt{a b}} \frac{1}{2 \pi i} \int_{(\varepsilon)} X_{+}(s)\left(\frac{q}{\pi}\right)^{s} \\
\sum_{m} m^{-(1 / 2+\alpha+s)} W\left(\frac{m}{M}\right) \int x^{-(1 / 2+\beta+s)} W\left(\frac{x}{N}\right) d x \frac{d s}{s} .
\end{aligned}
$$

Recall from (4.7) that

$$
\int_{0}^{\infty} x^{u-1} W\left(\frac{x}{N}\right) d x=\sum_{n} n^{u-1} W\left(\frac{n}{N}\right)+O_{\varepsilon}\left(q^{\varepsilon} N^{\operatorname{Re}(u)-1}|u|\right) .
$$

The contribution of the $O$-term to $\mathcal{M}_{2,<}(M, N)$ is $O_{\varepsilon}\left(q^{-1+\varepsilon} \sqrt{A B M / N}\right)$. Hence

$$
\mathcal{M}_{2,<}(M, N)=\frac{2}{q} \sum_{\substack{a, b \\ m, n}} \frac{\boldsymbol{\alpha}_{a} \boldsymbol{\beta}_{b}}{\sqrt{a b} m^{1 / 2+\alpha} n^{1 / 2+\beta}} W\left(\frac{m}{M}\right) W\left(\frac{n}{N}\right) V_{+}\left(\frac{\pi m n}{q}\right)+O_{\varepsilon}\left(q^{-1 / 2-4 \delta_{0}+\varepsilon}\right) .
$$

The same expression holds for $\mathcal{M}_{2,>}(M, N)$.

4.5. Assembling the partition of unity. Recall that Proposition 4.1 holds when $(M, N) \in \mathcal{A}_{3}$ and (4.10) holds when $(M, N) \in \mathcal{A}_{2, \gtrless}$. Summing up we obtain

$$
\begin{aligned}
S_{\alpha, \beta}^{+}= & \sum_{(M, N) \in \mathcal{A}_{3}}\left(\mathcal{P}_{1}^{\prime \prime}(M, N)+\mathcal{P}_{2}^{\prime \prime}(M, N)\right) \\
& +\sum_{(M, N) \notin \mathcal{A}_{1}} \frac{2}{q} \sum_{\substack{a, b \\
m, n}} \frac{\boldsymbol{\alpha}_{a} \boldsymbol{\beta}_{b}}{\sqrt{a b} m^{1 / 2+\alpha} n^{1 / 2+\beta}} W\left(\frac{m}{M}\right) W\left(\frac{n}{N}\right) V_{+}\left(\frac{\pi m n}{q}\right)+O_{\varepsilon}\left(q^{-\delta_{0}+\varepsilon}\right)
\end{aligned}
$$

for some $\delta_{0}>0$.

The condition $(M, N) \notin \mathcal{A}_{1}$ in the second sum may be removed at the cost of an error of size $O_{\varepsilon}\left(q^{-\delta_{0}+\varepsilon}\right)$. This allows us to extend the summation over all $(M, N)$, and thus to remove the partition of unity. For the first sum, the following result shall allow us to add all the missing pairs $(M, N)$.

Lemma 4.2. With $\mathcal{P}_{j}^{\prime \prime}(M, N), j=1,2$, defined as in (4.9) we have

$$
\mathcal{P}_{j}^{\prime \prime}(M, N) \ll_{\varepsilon} q^{\varepsilon} \min \left\{\frac{\sqrt{A B M N}}{q}, \sqrt{\frac{A}{N}}, \sqrt{\frac{B}{M}}\right\} .
$$

Proof. Recall from (4.3) and (4.4) that for $j=1,2, H_{j}$ 's have rapid decay as any of the variables gets large in the imaginary directions. So we have, trivially,

$$
\mathcal{P}_{j}^{\prime \prime}(M, N) \ll_{\varepsilon} q^{-\left(\sigma+c_{1}+c_{2}\right)+\varepsilon} M^{c_{1}} N^{c_{2}} A^{\sigma+c_{1}} B^{\sigma+c_{2}},
$$

provided that

$$
0<2 \sigma+c_{1}+c_{2}<1, \quad \sigma+c_{1}<\frac{1}{2} \quad \text { and } \quad \sigma+c_{2}<\frac{1}{2} .
$$

The lemma follows by choosing various suitable values of $\sigma, c_{1}$ and $c_{2}$. 
The above lemma implies that for $j=1,2$,

$$
\sum_{(M, N) \in \mathcal{A}_{3}} \mathcal{P}_{j}^{\prime \prime}(M, N)=\sum_{M, N} \mathcal{P}_{j}^{\prime \prime}(M, N)+O_{\varepsilon}\left(q^{-\delta_{0}+\varepsilon}\right) .
$$

Now we can apply the following result of Young [22; p. 30].

Lemma 4.3. Let $F\left(s_{1}, s_{2}\right)$ be an entire function of rapid decay in each variable in a fixed strip $\left|\operatorname{Re}\left(s_{j}\right)\right| \leq C, j=1,2$. Then we have

$$
\sum_{M, N} \frac{1}{(2 \pi i)^{2}} \int_{\left(c_{2}\right)} \int_{\left(c_{1}\right)} F\left(s_{1}, s_{2}\right) \widetilde{W}\left(s_{1}\right) \widetilde{W}\left(s_{2}\right) d s_{1} d s_{2}=F(0,0) .
$$

In view of this we get

$$
\sum_{(M, N) \in \mathcal{A}_{3}}\left(\mathcal{P}_{1}^{\prime \prime}(M, N)+\mathcal{P}_{2}^{\prime \prime}(M, N)\right)=\mathcal{N}_{\alpha, \beta}^{+}+O_{\varepsilon}\left(q^{-\delta_{0}+\varepsilon}\right)
$$

where

$$
\mathcal{N}_{\alpha, \beta}^{+}=\sum_{\substack{d \\(a, b)=1}} \frac{\boldsymbol{\alpha}_{d a} \boldsymbol{\beta}_{d b}}{d a b} \frac{1}{2 \pi i} \int_{(\varepsilon)} X_{+}(s) H(s)\left(\frac{q}{\pi}\right)^{s}\left(\frac{q}{a}\right)^{-(\alpha+s)}\left(\frac{q}{b}\right)^{-(\beta+s)} \zeta(\alpha+\beta+2 s) \frac{d s}{s}
$$

and

$$
\begin{gathered}
H(s)=\frac{\Gamma(\alpha+\beta+2 s) \Gamma(1 / 2-\beta-s)}{\Gamma(1 / 2+\alpha+s)}+\frac{\Gamma(\alpha+\beta+2 s) \Gamma(1 / 2-\alpha-s)}{\Gamma(1 / 2+\beta+s)} \\
+\frac{\Gamma(1 / 2-\alpha-s) \Gamma(1 / 2-\beta-s)}{\Gamma(1-\alpha-\beta-2 s)} .
\end{gathered}
$$

Using Lemma 8.2 of $[\mathbf{2 2}]$ we have

$$
H(s)=\pi^{1 / 2} \frac{\Gamma\left(\frac{\alpha+\beta+2 s}{2}\right) \Gamma\left(\frac{1 / 2-\alpha-s}{2}\right) \Gamma\left(\frac{1 / 2-\beta-s}{2}\right)}{\Gamma\left(\frac{1-\alpha-\beta-2 s}{2}\right) \Gamma\left(\frac{1 / 2+\alpha+s}{2}\right) \Gamma\left(\frac{1 / 2+\beta+s}{2}\right)} .
$$

So

$$
\begin{aligned}
\mathcal{N}_{\alpha, \beta}^{+}=\frac{\pi^{1 / 2} q^{-(\alpha+\beta)}}{\Gamma\left(\frac{1 / 2+\alpha}{2}\right) \Gamma\left(\frac{1 / 2+\beta}{2}\right)} \sum_{\substack{d \\
(a, b)=1}} \frac{\boldsymbol{\alpha}_{d a} \boldsymbol{\beta}_{d b}}{d a^{1-\alpha} b^{1-\beta}} \\
\\
\quad \frac{1}{2 \pi i} \int_{(\varepsilon)} G(s) \frac{\Gamma\left(\frac{\alpha+\beta+2 s}{2}\right) \Gamma\left(\frac{1 / 2-\alpha-s}{2}\right) \Gamma\left(\frac{1 / 2-\beta-s}{2}\right)}{\Gamma\left(\frac{1-\alpha-\beta-2 s}{2}\right)}\left(\frac{a b}{\pi q}\right)^{s} \zeta(\alpha+\beta+2 s) \frac{d s}{s} .
\end{aligned}
$$

We apply the functional equation,

$$
\begin{aligned}
\pi^{-(\alpha+\beta+2 s) / 2} & \Gamma\left(\frac{\alpha+\beta+2 s}{2}\right) \zeta(\alpha+\beta+2 s) \\
& =\pi^{-(1-\alpha-\beta-2 s) / 2} \Gamma\left(\frac{1-\alpha-\beta-2 s}{2}\right) \zeta(1-\alpha-\beta-2 s),
\end{aligned}
$$

and change the variable $s \rightarrow-s$ to obtain

$$
\mathcal{N}_{\alpha, \beta}^{+}=-\left(\frac{q}{\pi}\right)^{-(\alpha+\beta)} \sum_{\substack{d \\(a, b)=1}} \frac{\boldsymbol{\alpha}_{d a} \boldsymbol{\beta}_{d b}}{d a^{1-\alpha} b^{1-\beta}} \frac{1}{2 \pi i} \int_{(-\varepsilon)} X_{-}(s)\left(\frac{\pi a b}{q}\right)^{-s} \zeta(1-\alpha-\beta+2 s) \frac{d s}{s}
$$


We conclude that

Proposition 4.4. We have

$$
S_{\alpha, \beta}^{+}=\mathcal{N}_{\alpha, \beta}^{+}+\frac{2}{q} \sum_{\substack{a, b \\ m, n}} \frac{\boldsymbol{\alpha}_{a} \boldsymbol{\beta}_{b}}{\sqrt{a b} m^{1 / 2+\alpha} n^{1 / 2+\beta}} V_{+}\left(\frac{\pi m n}{q}\right)+O_{\varepsilon}\left(q^{-\delta_{0}+\varepsilon}\right),
$$

where $\mathcal{N}_{\alpha, \beta}^{+}$is defined as in (4.11).

4.6. Combining the main terms and secondary main terms. Section 2 and Proposition 4.4 imply that

$$
I_{\alpha, \beta}=\left(\mathcal{M}_{\alpha, \beta}^{+}+\mathcal{N}_{\alpha, \beta}^{+}\right)+\left(\frac{q}{\pi}\right)^{-(\alpha+\beta)}\left(\mathcal{M}_{-\beta,-\alpha}^{-}+\mathcal{N}_{-\beta,-\alpha}^{-}\right)+O_{\varepsilon}\left(q^{-\delta_{0}+\varepsilon}\right),
$$

where $\mathcal{M}^{ \pm}, \mathcal{N}^{ \pm}$are defined as in (2.2) and (4.11). We have, for instance,

$$
\begin{aligned}
\left(\frac{q}{\pi}\right)^{-(\alpha+\beta)} \mathcal{M}_{-\beta,-\alpha}^{-}+\mathcal{N}_{\alpha, \beta}^{+}= & \left(\frac{q}{\pi}\right)^{-(\alpha+\beta)} \sum_{\substack{d a, d b \leq q^{\kappa} \\
(a, b)=1}} \frac{\boldsymbol{\alpha}_{d a} \boldsymbol{\beta}_{d b}}{d a^{1-\alpha} b^{1-\beta}} \\
& \frac{1}{2 \pi i}\left(\int_{(\varepsilon)}-\int_{(-\varepsilon)}\right) X_{-}(s)\left(\frac{\pi a b}{q}\right)^{-s} \zeta(1-\alpha-\beta+2 s) \frac{d s}{s} \\
= & \operatorname{Res}_{s=0} \\
= & X_{-}(0)\left(\frac{q}{\pi}\right)^{-(\alpha+\beta)} \zeta(1-\alpha-\beta) \sum_{\substack{d a, d b \leq q^{\kappa} \\
(a, b)=1}} \frac{\boldsymbol{\alpha}_{d a} \boldsymbol{\beta}_{d b}}{d a^{1-\alpha} b^{1-\beta}} .
\end{aligned}
$$

Note that the pole at $s=(\alpha+\beta) / 2$ of the zeta-function is cancelled by the function $G$. A similar expression holds for the combination of the other two terms, and Theorem 1.1 follows.

\section{Proofs of Theorems 1.2 And 1.3}

5.1. Proof of Theorem 1.2. We argue the same as in the proof of Theorem 1.1. The only difference is that we also apply Proposition 3.3 to $(4.1)$ : if $(M, N) \in \mathcal{A}_{1}$ we apply (3.1) of Proposition 3.1; if $(M, N) \in \mathcal{A}_{2,<} \cup \mathcal{A}_{2,>}$ we apply Proposition 3.2; and in the remaining case we apply Proposition 3.3. So it remains to check that the error term $\mathcal{E}(M, N)$ is acceptable when $(M, N) \in \mathcal{A}_{3}$.

As in Subsection 4.2, if $(M, N) \in \mathcal{A}_{3}$, then we may assume that $M, N \ll q^{\kappa+6 \delta_{0}}$. Without loss of generality, let us assume that $A M \ll B N$. Then from Proposition 3.3,

$$
\mathcal{E}(M, N) \ll_{\varepsilon} q^{-1+\varepsilon} A^{1 / 4} B^{3 / 2}(N / M)^{1 / 2}+q^{-3 / 4+\varepsilon} A^{1 / 4} B(N / M)^{1 / 4}\left(A_{1}+A_{2}\right)^{1 / 4} .
$$

Since $M \gg q^{2-2 \delta_{0}} / A B N$, it follows that $N / M \ll q^{-2+2 \delta_{0}} A B N^{2} \ll q^{-2+4 \kappa+14 \delta_{0}}$, and hence

$$
\mathcal{E}(M, N) \ll_{\varepsilon} q^{-2+15 \kappa / 4+7 \delta_{0}+\varepsilon}+q^{-5 / 4+9 \kappa / 4+7 \delta_{0} / 2+\varepsilon}\left(A_{1}+A_{2}\right)^{1 / 4} .
$$

Thus $\mathcal{E}(M, N) \ll_{\varepsilon} q^{-\delta_{0}+\varepsilon}$ for some $\delta_{0}>0$ if $9 \kappa+\max \left\{\kappa_{1}, \kappa_{2}\right\}<5$. 
5.2. Proof of Theorem 1.3. Again we follow the arguments in the proof of Theorem 1.1. The only difference is that if $(M, N) \in \mathcal{A}_{3}$, then we apply Proposition 3.4 to (4.1). We only need to verify that the error term $\mathcal{E}(M, N)$ is negligible in this case.

As above, we may assume that $M, N \ll q^{\kappa+6 \delta_{0}}$, and, without loss of generality, that $A M \ll B N$. In view of Proposition 3.4,

$$
\mathcal{E}(M, N) \ll_{\varepsilon} q^{\vartheta-1 / 2+\varepsilon}(B N / A M)^{3 / 4}\left(A_{2} B_{2}+\frac{B^{2} N}{q M}\right)^{1 / 2}\left(A_{2} B_{2}+\frac{B}{A_{1}}\right)^{1 / 2}
$$

with $\vartheta=7 / 64$. Since $M \gg q^{2-2 \delta_{0}} / A B N$, we get

$$
\mathcal{E}(M, N) \ll_{\varepsilon} q^{\vartheta-2+3 \kappa / 2+21 \delta_{0} / 2+\varepsilon} B^{3 / 2}\left(A_{2} B_{2}+q^{-3+2 \kappa+14 \delta_{0}} A B^{3}\right)^{1 / 2}\left(A_{2} B_{2}+\frac{B}{A_{1}}\right)^{1 / 2} .
$$

As $M N \ll q^{1+\varepsilon}$, we have $A B \gg q^{1-2 \delta_{0}-\varepsilon}$, and so $B / A_{1} \ll q^{-1+2 \delta_{0}+\varepsilon} A_{2} B^{2}$. Hence

$$
\begin{aligned}
\mathcal{E}(M, N) & \ll_{\varepsilon} q^{\vartheta-2+3 \kappa+2 \kappa_{2}+21 \delta_{0} / 2+\varepsilon}+q^{\vartheta-5 / 2+4 \kappa+3 \kappa_{2} / 2+23 \delta_{0} / 2+\varepsilon}+q^{\vartheta-7 / 2+6 \kappa+\kappa_{2}+35 \delta_{0} / 2+\varepsilon} \\
& \ll_{\varepsilon} q^{\vartheta-1 / 2+7 \kappa_{2}+35 \delta_{0} / 2+\varepsilon} .
\end{aligned}
$$

Thus $\mathcal{E}(M, N) \ll_{\varepsilon} q^{-\delta_{0}+\varepsilon}$ for some $\delta_{0}>0$ if $\kappa_{2}<1 / 14-\vartheta / 7$.

\section{Proof of Theorem 1.4}

The lower bound for (1.3) is relatively straightforward, and follows from the work of Rudnick and Soundararajan [19]. We therefore focus on the upper bound. Our approach utilizes a combination of ideas from Heath-Brown [10] and Bettin, Chandee and Radziwiłł [2], as well as our Theorem 1.1 on the twisted second moment of Dirichlet $L$-functions. Heath-Brown [10; Theorem 1] previously obtained Theorem 1.4 assuming the Generalized Riemann Hypothesis, and Bettin, Chandee and Radziwiłł [2; Corollary 2] obtained the analogue of Theorem 1.4 for the Riemann zeta-function $\zeta(s)$.

Let us define

$$
M(q)=\sum_{\chi(\bmod q)}^{*}\left|L\left(\frac{1}{2}, \chi\right)\right|^{3},
$$

so that the upper bound in (1.3) follows from the estimate

$$
M(q) \ll q(\log q)^{9 / 4} .
$$

We follow Heath-Brown (see [10; p. 408-409]) and first obtain an upper bound for $M(q)$ in terms of an integral.

As $L(s, \chi)$ is an analytic function, we have

$$
\left|L\left(\frac{1}{2}, \chi\right)\right|^{3} \leq \frac{1}{2 \pi} \int_{0}^{2 \pi}\left|L\left(\frac{1}{2}+r e^{i \theta}, \chi\right)\right|^{3} d \theta
$$

for any $r \geq 0$. We multiply both sides of (6.1) by $r$ and integrate from 0 to $R$, obtaining

$$
\left|L\left(\frac{1}{2}, \chi\right)\right|^{3} \leq \frac{1}{\operatorname{meas}(D)} \int_{D}\left|L\left(\frac{1}{2}+z, \chi\right)\right|^{3} d A
$$

where $D=\{z:|z| \leq R\}$ and $d A$ denotes the area measure. We choose $R=(\log q)^{-1}$, and then the real part of $1 / 2+z$ satisfies

$$
\frac{1}{2}-\frac{1}{\log q} \leq \operatorname{Re}\left(\frac{1}{2}+z\right) \leq \frac{1}{2}+\frac{1}{\log q} .
$$


Now define a function

$$
W_{\rho}(s)=W(s)=\frac{q^{\rho(s-1 / 2)}-1}{(s-1 / 2) \log q},
$$

where $\rho>0$ is a parameter at our disposal (we eventually take $\rho$ to be rather small). For $z \in D$ we have

$$
\left|W\left(\frac{1}{2}+z\right)\right| \geq \frac{\rho}{2}
$$

provided that $\rho \leq 1 / 2$, say. By positivity, we obtain from (6.2) and (6.3) that

$$
\begin{aligned}
\left|L\left(\frac{1}{2}, \chi\right)\right|^{3} & \ll_{\rho}(\log q)^{2} \int_{D}\left|W\left(\frac{1}{2}+z\right)\right|^{6}\left|L\left(\frac{1}{2}+z, \chi\right)\right|^{3} d A \\
& \leq(\log q)^{2} \int_{-1 / \log q}^{1 / \log q} \int_{-\infty}^{\infty}\left|W\left(\frac{1}{2}+\gamma+i t\right)\right|^{6}\left|L\left(\frac{1}{2}+\gamma+i t, \chi\right)\right|^{3} d t d \gamma .
\end{aligned}
$$

We define

$$
J(\gamma)=\sum_{\chi(\bmod q)}^{*} \int_{-\infty}^{\infty}\left|W\left(\frac{1}{2}+\gamma+i t\right)\right|^{6}\left|L\left(\frac{1}{2}+\gamma+i t, \chi\right)\right|^{3} d t
$$

We have therefore obtained

$$
M(q) \ll_{\rho}(\log q)^{2} \int_{-1 / \log q}^{1 / \log q} J(\gamma) d \gamma .
$$

By $[\mathbf{1 0}$; Lemma 4] we have

$$
J(\gamma) \ll_{A} q(\log q)^{-1}+J\left(\frac{A}{\log q}\right),
$$

where $A \geq 1$ is a parameter at our disposal. Thus,

$$
M(q) \ll_{\rho, A} q+(\log q) J\left(\frac{A}{\log q}\right) .
$$

We shall eventually take $A$ to be a large, but fixed, constant.

In order to apply our main theorem, we need to truncate the integral in the definition of $J(\gamma)$ to $|t| \leq T$, for some relatively small $T$. This can be done easily because of the decay of $W$ in vertical strips. For $|t| \geq 1$ we have

$$
\left|W\left(\frac{1}{2}+\frac{A}{\log q}+i t\right)\right|^{6} \ll_{\rho, A} \frac{1}{t^{6}},
$$

so that

$$
\begin{gathered}
\sum_{\chi(\bmod q)}^{*} \int_{|t| \geq T}\left|W\left(\frac{1}{2}+\frac{A}{\log q}+i t\right)\right|^{6}\left|L\left(\frac{1}{2}+\frac{A}{\log q}+i t, \chi\right)\right|^{3} d t \\
\ll_{\rho, A} \sum_{\chi(\bmod q)}^{*} \int_{|t| \geq T}\left|L\left(\frac{1}{2}+\frac{A}{\log q}+i t, \chi\right)\right|^{3} \frac{d t}{t^{6}} .
\end{gathered}
$$

We break the integral into dyadic segments, so we must estimate

$$
\sum_{\chi(\bmod q)}^{*} \int_{U \leq|t| \leq 2 U}\left|L\left(\frac{1}{2}+\frac{A}{\log q}+i t, \chi\right)\right|^{3} \frac{d t}{t^{6}}
$$




$$
\ll \frac{1}{U^{6}} \sum_{\chi(\bmod q)}^{*} \int_{-2 U}^{2 U}\left|L\left(\frac{1}{2}+\frac{A}{\log q}+i t, \chi\right)\right|^{3} d t
$$

for $U \geq T$. By Hölder's inequality and Theorem 10.1 of [17], we find that this latter quantity is

$$
\ll \frac{(q U)^{1 / 4}}{U^{6}}\left(\sum_{\chi(\bmod q)}^{*} \int_{-2 U}^{2 U}\left|L\left(\frac{1}{2}+\frac{A}{\log q}+i t, \chi\right)\right|^{4} d t\right)^{3 / 4} \ll \frac{q(\log q U)^{3}}{U^{5}} .
$$

Summing over $U=2^{j}$ with $U \geq T=\log q$, we find that

$$
J\left(\frac{A}{\log q}\right)=J_{1}\left(\frac{A}{\log q}\right)+O_{\rho, A}\left(q(\log q)^{-1}\right)
$$

where

$$
J_{1}(\gamma)=\sum_{\chi(\bmod q)}^{*} \int_{-\log q}^{\log q}\left|W\left(\frac{1}{2}+\gamma+i t\right)\right|^{6}\left|L\left(\frac{1}{2}+\gamma+i t, \chi\right)\right|^{3} d t .
$$

We next require an upper bound for $L(1 / 2+A / \log q+i t, \chi)$. Here we mostly follow the arguments of $[2$; Section 6].

Lemma 6.1. Let $G$ be a compactly supported function. Suppose that $F(u)=-G^{\prime}(u)$ for $u>0$ and $F$ is three times continuously differentiable and compactly supported. Then

$$
\sum_{n} \frac{\chi(n)}{n^{s}} G\left(\frac{\log n}{\log x}\right)=\frac{1}{2 \pi i} \int_{(c)} L(s+w, \chi) \widehat{F}\left(\frac{-i w \log x}{2 \pi}\right) \frac{d w}{w},
$$

where $c>\max \{1-\operatorname{Re}(s), 0\}, x>1$ and $\widehat{F}$ denotes the Fourier transform of $F$.

Proof. Argue as in [2; Lemma 2].

Let $\delta>0$ be a small positive parameter to be chosen later. We introduce another parameter $\theta$ which satisfies $\delta<\theta<1$. We define

$$
\widehat{F}(z)=e^{2 \pi i(\theta-\delta) z}\left(\frac{e^{2 \pi i(1-\theta) z}-1}{2 \pi i(1-\theta) z}\right)^{N}
$$

where $N \geq 10$ is a bounded integer (actually, $N=10$ suffices). Then $F$ is compactly supported on $[\theta-\delta, \theta-\delta+(1-\theta) N]$. For $u>0$, we define

$$
G(u)=1-\int_{0}^{u} F(v) d v,
$$

and $G(u)=0$ for $u \leq-1$. We let $G$ decay smoothly to zero on the interval $[-1,0]$. Thus $F(u)=-G^{\prime}(u)$ for all $u>0$. We have $G(u)=1$ for $0<u<\theta-\delta$, and $G(u)=0$ for $u>\theta-\delta+(1-\theta) N$. Lastly, $G$ is $N$ times differentiable, and therefore $\widehat{G}(u) \ll(1+|u|)^{-N}$.

We now let $x \leq q^{1 / 2+1 / 300}$, and choose our parameters $\delta, \theta$ so that $\delta=2(N-1)(1-\theta)$, and $\theta=(\log y) /(\log x)$ with $y=q^{1 / 2+2 \delta}$. Note that we want $\delta$ to be small enough so that $x \leq q^{1 / 2+1 / 300}$. We remark that we shall eventually choose our parameter $\rho$ to be sufficiently small in terms of $\delta$. 
With such choice of $\delta$ and $\theta$ we have

$$
\widehat{F}\left(\frac{-i w \log x}{2 \pi}\right)=\left(y x^{-\delta}\right)^{w}\left(\frac{(x / y)^{w}-1}{w(1-\theta)(\log x)}\right)^{N} \text {. }
$$

Using Lemma 6.1 with $s=\sigma+i t$ and $\sigma=1 / 2+A / \log q$, we shift the line of integration to $\operatorname{Re}(w)=1 / 2-\sigma$, thereby obtaining

$$
|L(s, \chi)| \leq\left|\sum_{n} \frac{\chi(n)}{n^{s}} G\left(\frac{\log n}{\log x}\right)\right|+(4 N)^{N} \frac{\left(y x^{-\delta}\right)^{1 / 2-\sigma}}{(\delta \log x)^{N}} \int_{-\infty}^{\infty} \frac{\left|L\left(\frac{1}{2}+i t+i v, \chi\right)\right|}{\left((\sigma-1 / 2)^{2}+v^{2}\right)^{(N+1) / 2}} d v .
$$

Let

$$
c(n)=\sum_{\substack{n=a b \\ a, b \leq x}} d_{1 / 2}(a) d_{1 / 2}(b),
$$

where $d_{1 / 2}(n)$ are the Dirichlet coefficients of $\zeta(s)^{1 / 2}$. Then $c(n)=1$ for $n \leq x$. Since $G(u)=0$ for $u>1$, we obtain by Fourier inversion

$$
\begin{aligned}
\sum_{n} \frac{\chi(n)}{n^{s}} G\left(\frac{\log n}{\log x}\right) & =\sum_{n} \frac{c(n) \chi(n)}{n^{s}} G\left(\frac{\log n}{\log x}\right) \\
& =\frac{\log x}{2 \pi} \int_{-\infty}^{\infty}\left(\sum_{a \leq x} \frac{d_{1 / 2}(a) \chi(a)}{a^{s+i v}}\right)^{2} \widehat{G}\left(\frac{v \log x}{2 \pi}\right) d v .
\end{aligned}
$$

We then multiply both sides of (6.7) by $|W(s)|^{6}|L(s, \chi)|^{2}$, integrate over $t$, sum on $\chi$, apply (6.6) and (6.8) to obtain

$$
J_{1}\left(\frac{A}{\log q}\right) \leq \mathcal{M}+\mathcal{E}
$$

where

$$
\begin{aligned}
\mathcal{M}=\frac{\log x}{2 \pi} \int_{-\log q}^{\log q} \int_{-\infty}^{\infty}\left|\widehat{G}\left(\frac{v \log x}{2 \pi}\right)\right|\left|W\left(\frac{1}{2}+\frac{A}{\log q}+i t\right)\right|^{6} \\
\sum_{\chi(\bmod q)}^{*}\left|L\left(\frac{1}{2}+\frac{A}{\log q}+i t, \chi\right)\right|^{2}\left|\sum_{a \leq x} \frac{d_{1 / 2}(a) \chi(a)}{a^{1 / 2+A / \log q+i(t+v)}}\right|^{2} d v d t
\end{aligned}
$$

and

$$
\begin{gathered}
\mathcal{E}=(4 N)^{N} \frac{\left(y x^{-\delta}\right)^{-A / \log q}}{(\delta \log x)^{N}} \int_{-\infty}^{\infty} \int_{-\infty}^{\infty} \frac{|W(1 / 2+A / \log q+i t)|^{6}}{\left(A^{2} /(\log q)^{2}+v^{2}\right)^{(N+1) / 2}} \\
\sum_{\chi(\bmod q)}^{*}\left|L\left(\frac{1}{2}+\frac{A}{\log q}+i t, \chi\right)\right|^{2}\left|L\left(\frac{1}{2}+i t+i v, \chi\right)\right| d v d t .
\end{gathered}
$$

Observe that we have used positivity to extend the $t$-integral in $\mathcal{E}$ to all of $\mathbb{R}$. Our goal now is to show that

$$
\mathcal{M} \ll_{\rho, A, \delta} q(\log q)^{5 / 4}
$$

and

$$
\mathcal{E} \leq \frac{1}{2} J\left(\frac{A}{\log q}\right)
$$


Then (6.5), (6.9), (6.10) and (6.11) together give

$$
J_{1}\left(\frac{A}{\log q}\right) \ll_{\rho, A, \delta} q(\log q)^{5 / 4},
$$

and comparison with (6.4) and (6.5) leads to

$$
M(q) \ll_{\rho, A, \delta} q(\log q)^{9 / 4},
$$

as desired.

The bound (6.10) follows from the observation that

$$
\int_{-\log q}^{\log q}\left|W\left(\frac{1}{2}+\frac{A}{\log q}+i t\right)\right|^{6} d t \ll_{\rho, A}(\log q)^{-1}
$$

and the following lemma.

Lemma 6.2. Let $t, v \in \mathbb{R}$ with $|t| \leq \log q$, and $s=1 / 2+A / \log q+i t$. Then

$$
\sum_{\chi(\bmod q)}^{*}|L(s, \chi)|^{2}\left|\sum_{a \leq x} \frac{d_{1 / 2}(a) \chi(a)}{a^{s+i v}}\right|^{2} \ll_{A} q(\log q)^{9 / 4},
$$

and the implied constant is independent of $t$ and $v$.

Proof. Applying Theorem 1.1 with

$$
\alpha=\frac{A}{\log q}+i t, \quad \beta=\frac{A}{\log q}-i t \quad \text { and } \quad \boldsymbol{\alpha}_{a}=\frac{d_{1 / 2}(a)}{a^{A / \log q+i(t+v)}},
$$

the quantity on the left side of (6.12) is

$$
\ll_{A} q(\log q) \sum_{a, b \leq x} \frac{d_{1 / 2}(a) d_{1 / 2}(b)}{[a, b]}+O_{\varepsilon}\left(q^{1-\varepsilon}\right) .
$$

Note we have used the fact that $d_{1 / 2}(n) \geq 0$. By an Euler product computation we find

$$
\begin{aligned}
\sum_{a, b \leq x} \frac{d_{1 / 2}(a) d_{1 / 2}(b)}{[a, b]} & \leq \prod_{p \leq x}\left(\sum_{i, j \geq 0} \frac{d_{1 / 2}\left(p^{i}\right) d_{1 / 2}\left(p^{j}\right)}{\left[p^{i}, p^{j}\right]}\right)=\prod_{p \leq x}\left(1+\frac{5}{4 p}+O\left(\frac{1}{p^{2}}\right)\right) \\
& \ll(\log x)^{5 / 4} \ll(\log q)^{5 / 4},
\end{aligned}
$$

and the proof is complete.

We now proceed to show (6.11). For notational simplicity we write

$$
\mathcal{F}=(4 N)^{N} \frac{\left(y x^{-\delta}\right)^{-A / \log q}}{(\delta \log x)^{N}} .
$$

We make the change of variables $z=t+v$, obtaining

$$
\begin{aligned}
\mathcal{E}=\mathcal{F} \int_{-\infty}^{\infty} \int_{-\infty}^{\infty} \frac{|W(1 / 2+A / \log q+i t)|^{6}}{\left(A^{2} /(\log q)^{2}+(z-t)^{2}\right)^{(N+1) / 2}} & \\
& \sum_{\chi(\bmod q)}^{*}\left|L\left(\frac{1}{2}+\frac{A}{\log q}+i t, \chi\right)\right|^{2}\left|L\left(\frac{1}{2}+i z, \chi\right)\right| d z d t .
\end{aligned}
$$

By Hölder's inequality we obtain

$$
\mathcal{E} \leq \mathcal{F} \mathcal{E}_{1}^{2 / 3} \mathcal{E}_{2}^{1 / 3}
$$


where

$$
\begin{gathered}
\mathcal{E}_{1}=\sum_{\chi(\bmod q)}^{*} \int_{-\infty}^{\infty}\left|W\left(\frac{1}{2}+\frac{A}{\log q}+i t\right)\right|^{6}\left|L\left(\frac{1}{2}+\frac{A}{\log q}+i t, \chi\right)\right|^{3} \\
\int_{-\infty}^{\infty} \frac{d z}{\left(A^{2} /(\log q)^{2}+(z-t)^{2}\right)^{(N+1) / 2}} d t
\end{gathered}
$$

and

$$
\mathcal{E}_{2}=\sum_{\chi(\bmod q)}^{*} \int_{-\infty}^{\infty}\left|L\left(\frac{1}{2}+i z, \chi\right)\right|^{3} \int_{-\infty}^{\infty} \frac{|W(1 / 2+A / \log q+i t)|^{6}}{\left(A^{2} /(\log q)^{2}+(z-t)^{2}\right)^{(N+1) / 2}} d t d z .
$$

It is easy to bound $\mathcal{E}_{1}$. By changing variables we obtain

$$
\int_{-\infty}^{\infty} \frac{d z}{\left(A^{2} /(\log q)^{2}+(z-t)^{2}\right)^{(N+1) / 2}}=\left(\frac{\log q}{A}\right)^{N} \int_{-\infty}^{\infty} \frac{d u}{\left(1+u^{2}\right)^{(N+1) / 2}} \leq\left(\frac{\log q}{A}\right)^{N}
$$

and therefore

$$
\mathcal{E}_{1} \leq\left(\frac{\log q}{A}\right)^{N} J\left(\frac{A}{\log q}\right)
$$

Let us turn to $\mathcal{E}_{2}$. A change of variables yields

$$
\begin{gathered}
\int_{-\infty}^{\infty} \frac{|W(1 / 2+A / \log q+i t)|^{6}}{\left(A^{2} /(\log q)^{2}+(z-t)^{2}\right)^{(N+1) / 2}} d t \\
=\left(\frac{\log q}{A}\right)^{N} \int_{-\infty}^{\infty} \frac{|W(1 / 2+A / \log q+i z+i A u / \log q)|^{6}}{\left(1+u^{2}\right)^{(N+1) / 2}} d u
\end{gathered}
$$

We wish to replace the argument in $W$ by $1 / 2+A / \log q+i z$. Thus, we examine the quotient

$$
\begin{aligned}
& \frac{|W(1 / 2+A / \log q+i z+i A u / \log q)|}{|W(1 / 2+A / \log q+i z)|} \\
& \quad=\left|\frac{q^{\rho(A / \log q+i z+i A u / \log q)}-1}{q^{\rho(A / \log q+i z)}-1}\right|\left|\frac{A / \log q+i z}{A / \log q+i z+i A u / \log q}\right| .
\end{aligned}
$$

If $A \geq A_{0}(\rho)$, then

$$
\left|\frac{q^{\rho(A / \log q+i z+i A u / \log q)}-1}{q^{\rho(A / \log q+i z)}-1}\right| \ll 1 .
$$

Also, by considering the two cases $|A u / \log q-z| \geq|z| / 3$ and $|A u / \log q-z| \leq|z| / 3$, say, we find that

$$
\left|\frac{A / \log q+i z}{A / \log q+i z+i A u / \log q}\right| \ll 1+|u|
$$

and hence

$$
\begin{aligned}
\int_{-\infty}^{\infty} & \frac{|W(1 / 2+A / \log q+i z+i A u / \log q)|^{6}}{\left(1+u^{2}\right)^{(N+1) / 2}} d u \\
& \ll\left|W\left(\frac{1}{2}+\frac{A}{\log q}+i z\right)\right|^{6} \int_{-\infty}^{\infty} \frac{(1+|u|)^{6}}{\left(1+u^{2}\right)^{(N+1) / 2}} d u \ll\left|W\left(\frac{1}{2}+\frac{A}{\log q}+i z\right)\right|^{6} .
\end{aligned}
$$


We have therefore obtained

$$
\mathcal{E}_{2} \ll\left(\frac{\log q}{A}\right)^{N} \sum_{\chi(\bmod q)}^{*} \int_{-\infty}^{\infty}\left|W\left(\frac{1}{2}+\frac{A}{\log q}+i z\right)\right|^{6}\left|L\left(\frac{1}{2}+i z, \chi\right)\right|^{3} d z .
$$

The right side of (6.15) is similar to $J(0)$, but the argument of $W$ is perturbed. If we had precisely $J(0)$, we could apply [10; Lemma 4] to relate $J(0)$ to $J(A / \log q)$. We claim the bounds

$$
\begin{aligned}
\int_{-\infty}^{\infty} & \left|W\left(\frac{1}{2}+\frac{A}{\log q}+i z\right)\right|_{\chi(\bmod q)}^{6}\left|L\left(\frac{1}{2}+i z, \chi\right)\right|^{3} d z \\
& \ll e^{3 A / 2+O(\rho A)} \int_{-\infty}^{\infty}\left|W\left(\frac{1}{2}+\frac{2 A}{\log q}+i z\right)\right|_{\chi(\bmod q)}^{6}\left|L\left(\frac{1}{2}+\frac{A}{\log q}+i z, \chi\right)\right|^{3} d z \\
& \leq e^{3 A / 2+O(\rho A)} J\left(\frac{A}{\log q}\right) .
\end{aligned}
$$

The proof is very similar to the proof of [10; Lemma 4], so we do not give it. The only real difference is that we must do more careful bookkeeping with the constants. To prove (6.16) we also use the bounds

$$
\begin{aligned}
\left|W\left(\frac{1}{2}+\frac{2 A}{\log q}+i z\right)\right| & \ll e^{O(\rho A)}\left|W\left(\frac{1}{2}+\frac{A}{\log q}+i z\right)\right|, \\
\left|W\left(\frac{1}{2}+i z\right)\right| & \ll e^{O(\rho A)}\left|W\left(\frac{1}{2}+\frac{A}{\log q}+i z\right)\right|,
\end{aligned}
$$

which hold for $A \geq A_{0}(\rho)$. By (6.15) and (6.16) we therefore obtain

$$
\mathcal{E}_{2} \ll e^{3 A / 2+O(\rho A)}\left(\frac{\log q}{A}\right)^{N} J\left(\frac{A}{\log q}\right) .
$$

We compare (6.13), (6.14) and (6.17) and find

$$
\mathcal{E} \ll \mathcal{F} e^{A / 2+O(\rho A)}\left(\frac{\log q}{A}\right)^{N} J\left(\frac{A}{\log q}\right) .
$$

Recalling our definitions, we have

$$
\mathcal{E} \leq C e^{-\left(1+O\left(\rho \delta^{-1}\right)\right) \delta A}\left(\frac{8 N}{\delta A}\right)^{N} J\left(\frac{A}{\log q}\right)
$$

for some absolute constant $C>0$. We obtain (6.11) by choosing $\rho$ to be sufficiently small in terms of $\delta$, and then choosing $A$ to be sufficiently large in terms of $\rho$ and $\delta$.

\section{ACKNOWLEDGMENTS}

The second author was supported by NSF grant DMS-1501982. The authors would like to thank Sandro Bettin and Maksym Radziwiłł for various helpful comments. The authors would like to thank the referees for pointing out very useful clarifications and insights that have increased the quality of the manuscript. 


\section{REFERENCES}

[1] S. Bettin, V. Chandee, Trilinear forms with Kloosterman fractions, Adv. Math. 328 (2018), 12341262.

[2] S. Bettin, V. Chandee, M. Radziwiłt, The mean square of the product of the Riemann zeta-function with Dirichlet polynomials, J. Reine Angew. Math. 729 (2017), 51-79.

[3] H. M. Bui, Non-vanishing of Dirichlet L-functions at the central point, Int. J. Number Theory 8 (2012), 1855-1881.

[4] J. B. Conrey, H. Iwaniec, K. Soundararajan, The mean square of the product of a Dirichlet Lfunction and a Dirichlet polynomial, Funct. Approx. Comment. Math. 61 (2019), no. 2, 147-177.

[5] W. Duke, J. Friedlander, H. Iwaniec, Representations by the determinant and mean values of L-functions, Sieve methods, exponential sums, and their applications in number theory (Cardiff, 1995), London Math. Soc. Lecture Note Ser., vol. 237 (1997), 109-115.

[6] W. Duke, J. Friedlander, H. Iwaniec, Bilinear forms with Kloosterman fractions, Invent. Math. 128 (1997), 23-43.

[7] J. Friedlander, H. Iwaniec, A mean-value theorem for character sums, Michigan Math. J. 39 (1992), 153-159.

[8] I. S. Gradshteyn, I. M. Ryzhik, Table of integrals, series, and products, Academic Press, New York, (1965).

[9] W. Heap, M. Radziwitł, and K. Soundararajan, Sharp upper bounds for fractional moments of the Riemann zeta function, Q. J. Math. 70 (2019), no. 4, 1387-1396.

[10] D. R. Heath-Brown, Fractional moments of Dirichlet L-functions, Acta Arith. 145 (2010), 397409.

[11] D. R. Heath-Brown, C. Jia, The distribution of ap modulo one, Proc. London Math. Soc. 84 (2002), 79-104.

[12] B. Hough, The angle of large values of L-functions, J. Number Theory 167 (2016), 353-393.

[13] H. Iwaniec, E. Kowalski, Analytic Number Theory, American Mathematical Society Colloquium Publications, vol. 53, American Mathematical Society, Providence, RI (2004).

[14] H. Iwaniec, P. Sarnak, Dirichlet L-functions at the central point, Number Theory in Progress, vol. 2, de Gruyter, Berlin (1999), 941-952.

[15] R. Khan, H. Ngo, Nonvanishing of Dirichlet L-functions, Algebra Number Theory 10 (2016), 2081-2091.

[16] P. Michel, J. VanderKam, Non-vanishing of high derivatives of Dirichlet L-functions at the central point, J. Number Theory 81 (2000), 130-148.

[17] H. L. Montgomery, Topics in multiplicative number theory, Lecture Notes in Mathematics, vol. 227, Springer-Verlag, Berlin-New York (1971).

[18] M. Radziwiłl, K. Soundararajan, Moments and distribution of central L-values of quadratic twists of elliptic curves, Invent. Math. 202 (2015), 1029-1068.

[19] Z. Rudnick, K. Soundararajan, Lower bounds for moments of L-functions, Proc. Natl. Acad. Sci. USA 102 (2005), 6837-6838.

[20] K. Soundararajan, Mean-values of the Riemann zeta-function, Mathematika 42 (1995), 158-174.

[21] N. Watt, Bounds for a mean value of character sums, Int. J. Number Theory 4 (2008), 249-293.

[22] M. P. Young, The fourth moment of Dirichlet L-functions, Ann. of Math. 173 (2011), 1-50.

[23] R. Zacharias, Mollification of the fourth moment of Dirichlet L-functions, Acta Arith. 191 (2019), no. $3,201-257$.

Department of Mathematics, University of Manchester, Manchester M13 9PL, UK

E-mail address: hung.bui@manchester.ac.uk

Department of Mathematics, University of Illinois, 1409 West Green Street, UrBANA, IL 61801, USA

Current address: All Souls College, University of Oxford, UK

E-mail address: kyle.pratt@maths.ox.ac.uk 
Department of Mathematics, University of Illinois, 1409 West Green Street, UrBANA, IL 61801, USA

E-mail address: nirobles@illinois.edu

Department of Mathematics, University of Illinois, 1409 West Green Street, Urbana, IL 61801, USA and Simion Stoilow Institute of Mathematics of the Romanian ACAdemy, P.O. Box 1-764, RO-014700 Bucharest, Romania

E-mail address: zaharesc@illinois.edu 